\title{
Evaluation of Industry Standard Turbulence Models on an Axisymmetric Supersonic Compression Corner
}

\author{
James R. DeBonis* \\ NASA Glenn Research Center, Cleveland, Ohio 44135
}

\begin{abstract}
Reynolds-averaged Navier-Stokes computations of a shock-wave/boundary-layer interaction (SWBLI) created by a Mach 2.85 flow over an axisymmetric 30-degree compression corner were carried out. The objectives were to evaluate four turbulence models commonly used in industry, for SWBLIs, and to evaluate the suitability of this test case for use in further turbulence model benchmarking. The Spalart-Allmaras model, Menter's Baseline and Shear Stress Transport models, and a low-Reynolds number $k-\epsilon$ model were evaluated. Results indicate that the models do not accurately predict the separation location; with the SST model predicting the separation onset too early and the other models predicting the onset too late. Overall the Spalart-Allmaras model did the best job in matching the experimental data. However there is significant room for improvement, most notably in the prediction of the turbulent shear stress. Density data showed that the simulations did not accurately predict the thermal boundary layer upstream of the SWBLI. The effect of turbulent Prandtl number and wall temperature were studied in an attempt to improve this prediction and understand their effects on the interaction. The data showed that both parameters can significantly affect the separation size and location, but did not improve the agreement with the experiment. This case proved challenging to compute and should provide a good test for future turbulence modeling work.
\end{abstract}

\section{Nomenclature}

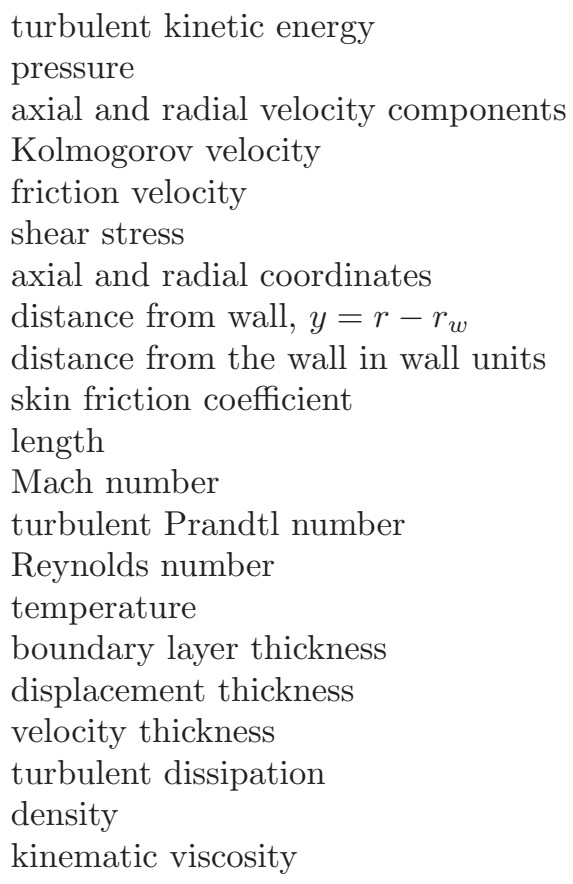

*Aerospace Engineer, Inlets \& Nozzles Branch, james.r.debonis@nasa.gov, Associate Fellow AIAA 
$\tilde{\nu}$

$\theta$

subscripts
$c$
reat
sep
w
0
$\infty$

Spalart-Allmaras turbulence variable

flare angle

cylinder

reattachment

separation

wall

stagnation condition

freestream condition

\section{Introduction}

After approximately two decades of neglect, research into turbulence modeling has been reinvigorated. During this time, improvements in Reynolds-averaged Navier-Stokes (RANS) computations can be attributed primarily to increases in computational power, which resulted in increased mesh sizes and reduced numerical error. Now, because we have adequate computational capacity for most conceivable RANS computations, we have come to the realization that further improvements in RANS must come from improvements in the underlying models. In addition, the computational fluid dynamics (CFD) community has looked towards scale resolving simulations such as large-eddy simulation (LES) and direct numerical simulation (DNS) as the next source of improvements in CFD. However, due to the enormous grid requirements for wall bounded flows at high Reynolds numbers, these methods will not be practical for decades to come. ${ }^{1}$ Hybrid RANS/LES methods are seen as bridging the gap to the future, but these methods will still be limited by the RANS turbulence modeling component. A recent NASA sponsored study, which projected the use of CFD to the year 2030, concluded that RANS analyses will still comprise a significant portion of all CFD analyses for the foreseeable future. ${ }^{2}$

RANS turbulence models perform well for attached boundary layers but fail in the presence of more complex flow physics such as adverse pressure gradients, large separated regions, and free shear flows. Shockwave/boundary-layer interactions (SWBLIs) are particularly challenging for RANS turbulence models due to the presence of shock waves, very large pressure gradients, and large separated zones. ${ }^{3}$ There has been great interest and numerous efforts over the years to improve the prediction of SWBLIs because they feature prominently in transonic and supersonic vehicles and propulsion systems. A recent example was the American Institute of Aeronautics and Astronautics (AIAA) Shock Boundary Layer Interaction Workshop; held in 2010 and summarized by Benek and Babinsky, ${ }^{4}$ and DeBonis et al. ${ }^{5}$ In this workshop, numerous researchers analyzed a set of common flows and shared their results and experiences. Two different flows were examined but both consisted of oblique shock waves impinging on a turbulent boundary layer along the bottom wall of a rectangular cross-sectioned wind tunnel. The interaction of the shock wave with the tunnel sidewalls induced complex three-dimensional corner flows that required large three-dimensional grids and long run-times, and further complicated an already difficult problem.

To address the current needs in predicting turbulent flows, NASA instituted the Revolutionary Computational Aerosciences (RCA) sub-project under the Aeronautical Sciences Project. RCA sponsors work in turbulence modeling, scale-resolving simulations, numerical methods, and transition prediction. In order to establish the current state-of-the-art in turbulence modeling and a baseline upon which to judge future modeling improvements, NASA is developing a set of standard test cases for representative flows of interest. Shock-wave/boundary-layer interaction is one of these flows. This paper examines a SWBLI based on an axisymmetric compression corner flow. The axisymmetric geometry and resulting flowfield greatly reduces the complexity of the analysis and flowfield while still retaining the key flow features. This case is being considered by the RCA sub-project for one of its standard test cases.

\section{Experimental Setup}

A series of tests on axisymmetric compression corners at supersonic Mach numbers were run at the NASA Ames Research Center in the 1980s. The test facility, the High Reynolds Number Facility - Channel 
I, was a blow-down tunnel with test times on the order of 16 minutes. The test article consisted of a $5.08 \mathrm{~cm}$ diameter body of revolution terminating with a 30-degree conical flare, which generated the shock wave (Fig. 1). The model had a cusped nose that was designed using the method of characteristics to minimize the initial shock wave. The length of the nose and cylinder preceding the flare was $1 \mathrm{~m}$. A turbulent boundary layer developed on this surface prior to the SWBLI. The flare surface begins at $x=0.000 \mathrm{~cm}$ and terminates at $x=5.196 \mathrm{~cm}$. The flow conditions of the test and subsequent analysis are given in Table 1 .

Table 1: Freestream conditions

\begin{tabular}{ll} 
Quantity & Value \\
\hline Reynolds number, Re $/ L$ & $16 \cdot 10^{6} / \mathrm{m}$ \\
Mach Number, $M$ & 2.85 \\
Stagnation pressure, $p_{0}$ & $1.7 \mathrm{~atm}$ \\
Stagnation temperature, $T_{0}$ & $270 \mathrm{~K}$
\end{tabular}

The data, taken over several experimental campaigns, consisted of schlieren photography, oil flow visualization, surface static pressures, holographic interferometry measurements of density, laser doppler velocimetry (LDV) ${ }^{6-8}$ and laser interferometric measurements of skin friction (LISF). ${ }^{9}$ The LDV data consists of two-component velocity measurements taken at 22 stations along the model and are presented as profiles of mean axial and radial velocity, and Reynolds stresses. The holographic interferometry data consists of static density profiles measured at 15 stations along the model.

The skin friction measurements were done on a variation of the geometry studied here, where a threedimensional SWBLI was generated by offsetting the centerline of the conical flare with respect to the cylinder. The upstream portion of the model and flow conditions remained the same, so we may use the upstream skin friction measurements, prior to the SWBLI, for comparison.

The experimentalist recommends using the final compilation of pressure, velocity, and density data found in Settles and Dodson ${ }^{10}$ for CFD comparisons. ${ }^{11}$ The skin friction data was published later and is taken from Wideman et al. ${ }^{9}$

\section{CFD Methodology}

\section{A. Grid}

A two-dimensional structured grid representing the body of revolution was generated using the Gridgen commercial mesh generation software. ${ }^{12}$ The grid (Fig. 2) begins on the body, just downstream of the cusped nose and approximately $75 \mathrm{~cm}$ from the start of the flare. The precise distance was altered until the CFD generated boundary layer profile matched the experimental profile at the first measurement station. The grid extends $10 \mathrm{~cm}$ beyond the downstream corner of the flare. The upper boundary is located $50 \mathrm{~cm}$ from the cylinder surface.

Several versions of the grid were made and analyzed before settling on the final version reported here. This was necessary in order to achieve grid independence. The final grid contains 922,185 points and could be considered extremely dense for a two-dimensional problem, relative to typical practice. This grid density was necessary to achieve grid independence for all the turbulence models examined in this study. The prediction of the shock location varies linearly with grid refinement, requiring fine grids in the vicinity of the shock. In addition the location and extent of the flow separation could be very sensitive to shock location and strength. These two factors resulted in solutions that varied with grid refinement much more than expected.

There are several features of this grid that should be noted.

- There are a total of 1,265 axial points and 729 radial points. There are 385 axial points located on the flare surface.

- Grid lines are orthogonal to the wall. This can be important for turbulence models where wall distance is used and computed along grid lines.

- Grid lines are parallel to, and clustered near the expected shock location. 
- The first grid point off the surface was located $5.0 \cdot 10^{-5} \mathrm{~cm}$ from the wall. This corresponded to $y^{+}=0.068$ at the furthest upstream velocity measurement station, prior to the interaction. In the interaction region and on flare surface this $y^{+}$value remained below 0.20 .

\section{B. Flow Solver}

The flow solver used for all the computations was the Wind-US code. ${ }^{13}$ Wind-US is a general purpose Reynolds-averaged Navier-Stokes equation solver with numerous options for numerical scheme, gas models, turbulence models, etc. All the calculations presented here used the same code options, listed below.

- axisymmetric mode

- full block implicit time stepping

- the OVERFLOW code's implementation of the Roe scheme, ${ }^{14}$ which includes an entropy fix to prevent expansion shocks

- minmod total variation diminishing limiter, with a compression parameter of 2

The inflow boundary is fixed using the freestream conditions. Conditions on the outflow boundary are extrapolated using first-order numerics. A characteristic-based freestream condition is applied to the upper boundary. No-slip adiabatic conditions are applied to the solid walls.

\section{Turbulence Models}

Four turbulence models were examined: the Spalart-Allmaras (SA) model, ${ }^{15}$ Menter's Baseline (BSL) and Shear Stress Transport (SST) models, ${ }^{16}$ and a low-Reynolds number $k-\epsilon$ model. ${ }^{17}$ These models are used for the vast majority of Reynolds-averaged Navier-Stokes computations in the aerospace industry, with the SA and SST models being the most common.

There are a few details on the implementation of the turbulence models that should be noted.

- The SA model uses the standard incompressible version without the trip term and the freestream value of $\tilde{\nu} / \nu$ is set to 5 .

- The SST and BSL models use the vorticity-based production term instead of the strain-rate based term.

- The $k-\epsilon$ model used here is the formulation of Abe, Kondoh, and Nagano ( $k-\epsilon$ AKN). This model was recently implemented in a developmental version of Wind-US and will be in the next publicly released version of the code.

The Chien $k-\epsilon$ formulation, ${ }^{18}$ which is in the current release version of Wind-US, was used for the initial low Reynolds number $k-\epsilon$ solutions. However, the model exhibited nonphysical behavior in the separated region. Chien's formulation uses friction velocity, $u_{\tau}$, in the near wall damping terms. Near the separation and reattachment locations $u_{\tau}$ becomes small, leading to small values of turbulent viscosity. The Abe, Kondoh, and Nagano formulation avoids problems in separated regions by replacing the friction velocity with the Kolmogorov velocity, $u_{\eta}$.

\section{Results}

A brief description of the flowfield will be given first. Mach number contours and a numerical shadowgraph, from the SA model solution, depict the complex shock-wave/boundary-layer interaction region (Fig. 3). A fully turbulent boundary layer exists upstream of the cylinder's flare. The flare surface creates the primary shock wave in the freestream flow. This shock wave separates the boundary layer in the corner between the cylinder and flare surfaces. The separation extends forward of the corner and the resultant thickened boundary layer induces a second shock wave forward of the corner. The flare surface turns the flow behind the separation shock wave causing a third shock wave that coalesces with the primary wave. The boundary layer reattaches to the flare approximately one-third of the way along the surface. An expansion wave forms at the downstream corner of the flare, where the flow turns back axially, and expands outward 
weakening the initial shock wave. These flow features agree well with the interferometery ${ }^{6}$ and schlieren ${ }^{8}$ images from the experiment.

Figure 4 is a very close-up view of the compression corner. The streamlines traveling in the $-x$-direction show a portion of the large separated region. In the corner, a small counter-rotating vortex is evident. A stagnation region exists between these two recirculating zones, as indicated by the relatively high levels of pressure seen in the contours.

\section{A. Grid Independence}

Grid sequencing was used to speed convergence and check grid independence. Solutions were obtained on a series of coarse (every 4th point used), medium (every other point used) and fine (every point used) grids. The solutions were interpolated onto the next finer grid and convergence was checked at each grid level in order to ensure an accurate assessment of grid independence. Excellent grid independence was achieved for all cases except for the SST model. Here, there were slight differences between the medium and fine grid solutions. However, the surface static pressures on the medium grid were at all points within one-percent of the fine grid result. As will be shown, the SST model produced the largest separated zone of all the models and it appears that this large separation is sensitive to the small movement of the shock position with grid refinement.

\section{B. Upstream Boundary Layer}

In order to properly simulate the experimental shock-wave/boundary-layer interaction, it is necessary to accurately match the simulation boundary layer to that of the experiment, upstream of the interaction. The experimental velocity and density profiles were not measured at the same locations. The furthest upstream location of each will be used; the velocity data is located at $x=-4.5 \mathrm{~cm}$ and the density data is located at $x=-5.03 \mathrm{~cm}$. In addition, the experimentalists reported a displacement thickness at the $x=-4.5 \mathrm{~cm}$ velocity measurement location.

As will be shown later, it was not possible to match both the velocity and density profiles for any of the CFD simulations. For this study the velocity boundary layer was matched. A velocity thickness was computed from the experimental velocity data at the upstream location. The velocity thickness is defined as

$$
\delta_{v}^{*}=\int_{r_{w}}^{r_{w}+\delta}\left(1-\frac{u}{u_{\infty}}\right) \frac{r}{r_{w}} d r
$$

The length of the cylinder upstream of the interaction region was varied in order to match this quantity for each simulation. Table 2 shows the computed velocity thicknesses and displacement thicknesses at the $x=-4.5 \mathrm{~cm}$ location, along with the corresponding lengths of the cylinder, for the four turbulence models. The BSL, $k-\epsilon$ AKN, and SA models all came within two percent of the experimental velocity thickness. The SST model solution separates prior to this first measurement station and results in a much higher value of $\delta_{v}^{*}$ than the experiment and other solutions. Because the SST solution is biased here, due to its separated state, a proper comparison of the boundary layer can not be done at this location. Since the SST and BSL models have very similar behavior in zero pressure gradient situations, they produce similar boundary layers further upstream of the the interaction. For this reason the cylinder length used for the BSL model was also used for the SST model. The poor predictions of displacement thickness, for all models, is due to poor predictions of the density profile. This will be discussed in further detail later.

\section{Comparison of Turbulence Models to Experimental Data}

\section{Static Pressure Distributions}

Surface static pressures are plotted in Fig. 5 for all four turbulence models. The pressure rise ahead of the compression corner $(x<0 \mathrm{~cm})$ is due to a shock wave induced by the separated boundary layer feeding forward of the flare. The large pressure rise further downstream $(0<x<5)$ is created by the shock waves induced by the flare surface. The pressure drops rapidly at the downstream corner as the flow expands here and reattaches. The small inflection in the pressure curves at $x=0 \mathrm{~cm}$ is the pressure rise in the stagnation region caused by the counter-rotating vortex in the compression corner (Fig. 4).

With the exception of the SST model, all the turbulence models show very similar static pressure distributions. The BSL, $k-\epsilon \mathrm{AKN}$, and SA models differ in the location and magnitude of the initial pressure rise 
Table 2: Upstream boundary layer state

\begin{tabular}{lcrrrr} 
& $\delta_{v}^{*}(\mathrm{~cm})$ & $\%$ error & $\delta^{*}(\mathrm{~cm})$ & $\%$ error & $L_{c}(\mathrm{~cm})$ \\
\hline Experiment, reported & & & 0.2965 & & \\
Experiment, computed & 0.1321 & & & & \\
BSL & 0.1320 & -0.0757 & 0.3367 & 13.5581 & 75.0 \\
SST & 0.1430 & 8.2513 & 0.3277 & 10.5228 & 75.0 \\
$k-\epsilon$ AKN & 0.1297 & -1.8168 & 0.3227 & 8.8364 & 78.0 \\
SA & 0.1305 & -1.2112 & 0.3375 & 13.8280 & 75.0
\end{tabular}

due to the separation location and size. The SA model shows the best agreement with the experiment. The SST model produces a separation bubble that feeds much further forward and the maximum pressure rise is diminished.

\section{Skin Friction Coefficient}

The exact location and extent of the separation can be obtained by examining the skin friction coefficient. Wideman measured skin friction using the LISF technique for a similar but three-dimensional shockwave/boundary-layer interaction and reported upstream values of $C_{f}$ ranging between 0.00155 and 0.0017 , depending on the surface treatment used. ${ }^{9}$ These bounds are included on the upstream portion of the plot. The experimentalists performed oil flow visualizations and reported the locations of the oil accumulations associated with the separation and reattachment points. The locations were digitized from Dunagan et al.: ${ }^{6}$ $x_{\text {sep }}=-2.73 \mathrm{~cm}$, and $x_{\text {reat }}=0.97 \mathrm{~cm}$. They caution that these locations are approximate and examination of the pressure distributions and velocity measurements will show that the separation point appears to be upstream of the oil flow derived location.

The skin friction coefficient is shown in Fig. 6. All models produce very similar skin frictions upstream of the interaction region, and they fall within the bounds of the experimental measurements. The separated region is evidenced by negative values of $C_{f}$. The location of the onset of separation in the skin friction data correlates very well with the initial pressure rise in Fig. 5. At the onset of separation, all CFD solutions predict a pressure increase of between 18.9 and 20.6 percent of the freestream value. The experimental data showed that the pressure rises between $x=-3.25$ and $x=-2.75$ centimeters, and the pressure rise is 34.2 percent of the freestream pressure at $x=-2.75 \mathrm{~cm}$. Using this information, we can infer that the experiment separated between $x=-3.25$ and $x=-2.75$ centimeters, upstream of the location identified in the oil flow visualization. The reattachment location can also be seen in Fig. 6. The BSL and $k-\epsilon$ AKN models show early reattachment locations. The SA and SST models predict later reattachment locations and a similar rise in $C_{f}$. The large spike in skin friction is at the aft end of the flare and is due to the acceleration of the flow around the corner. Similar to the pressure data, there is an inflection in $C_{f}$ at $x=0$. This positive region of skin friction, in the middle of the separated zone, is caused by the small counter-rotating vortex described previously and shown in Fig. 4.

The separation point, reattachment point, and separation length for the four models are listed in Table 3 . The SST model predicts an early separation that is 44 percent larger than the next largest prediction, the SA solution. The SA, SST, and BSL models all predict the onset of separation downstream of the experimental value. The separation length information shows that the current predictions follow the accepted trends reported in the literature; the SST model predicts the largest separations, the $k$ - $\epsilon$ model predicts the smallest separation, and the BSL and SA models predict intermediate sized separations.

\section{Velocity Data}

Velocity and Reynolds stress profiles were measured at 22 stations on the model between $x=-4.500 \mathrm{~cm}$ and $x=9.396 \mathrm{~cm}$. The data were taken normal to the cylinder axis. As a result, the profiles in the region of the flare are not normal to the flare surface. The CFD data were interpolated onto the experimental rake positions for comparison. Eight profiles are shown here: $x=-4.500, x=-3.000, x=-2.000, x=-1.000$, 
Table 3: Effect of turbulence model on the separated region

\begin{tabular}{|c|c|c|c|c|c|}
\hline & Wall Temp. (K) & $P r_{t}$ & $\begin{array}{c}\text { Separation } \\
\text { Location }(\mathrm{cm})\end{array}$ & $\begin{array}{l}\text { Reattachment } \\
\text { Location }(\mathrm{cm})\end{array}$ & $\begin{array}{l}\text { Separation } \\
\text { Length }(\mathrm{cm})\end{array}$ \\
\hline Experiment (oil flow) & & & -2.73 & 0.97 & 3.70 \\
\hline BSL & adiabatic & 0.9 & -2.5000 & 1.3849 & 3.8849 \\
\hline $\mathrm{SST}$ & adiabatic & 0.9 & -4.5657 & 2.5399 & 7.1056 \\
\hline$k-\epsilon$ & adiabatic & 0.9 & -2.2125 & 1.1156 & 3.3280 \\
\hline SA & adiabatic & 0.9 & -2.6750 & 2.2736 & 4.9236 \\
\hline
\end{tabular}

$x=-0.433, x=1.732, x=3.464$, and $x=5.896$ centimeters. These stations were selected to provide an even distribution of stations with emphasis on the separated zone.

Axial velocity profiles are shown in Fig. 7. The first profile $(x=-4.500 \mathrm{~cm})$ was at the upstream station used to match the velocity thickness and set the length of the cylinder in the computation. Here all models show good agreement with the boundary layer thickness. However, the experiment exhibits a fuller turbulent profile. The $k-\epsilon \mathrm{AKN}$ model displays a sharp change in curvature at the edge of the boundary layer. The SST model is already separated at this location, and its profile has lifted away from the wall.

Examining the downstream profiles shows that the solutions from all the models show poor agreement with the experimental data. In general, all the models show stronger reverse flow and a larger deficit near the wall in the separated region. The SST solution is the extreme example. The radial location of the shock can be seen in the outer portion of the velocity profiles. The larger separations predicted with SST and SA correspond to a more outboard location of the shock, as the larger volume of separated flow creates a larger flow deflection in the supersonic stream.

Radial velocity profiles are shown in Fig. 8. The experimental data at $x=-3.000 \mathrm{~cm}$ shows a vertical component to the flow in the boundary layer, suggesting that the experimental boundary layer was close to, or beginning to separate at this location. The radial velocity data shows that the CFD solutions all appear to show stronger recirculation, higher radial velocities, in the separated region. It also confirms the previous trends in terms of separation size and location, and outboard shock location.

Turbulent shear stress profiles are presented in Fig. 9. For the upstream profile at $x=-4.500 \mathrm{~cm}$, agreement for the attached solutions near the wall is poor, but good in the outer portion of the boundary layer. At $x=-3.000 \mathrm{~cm}$ the experiment again shows evidence that the flow is at or near separation, with larger values of shear away from the wall, while the BSL, $k-\epsilon$, and SA models remain attached. In the separated region, agreement with the data is very poor with all models disagreeing with the shape of the curves and even sign of the shear stress.

It must be noted that for some profiles $(x=0.433,1.732$ and $3.464 \mathrm{~cm})$ the CFD and experiment appear to produce similarly shaped curves with opposite signs. The possibility of a sign error was considered, but ruled out. Care was taken to ensure proper processing of the CFD solutions. To lend some credibility to the data presented, the sign of both the experimental and CFD shear stress is correct in the attached boundary layer profile at $x=-4.500 \mathrm{~cm}$, and all profiles were processed in the same manner.

\section{Density Data}

Density profiles were measured at 15 stations on the model between $x=-5.030 \mathrm{~cm}$ and $x=8.194 \mathrm{~cm}$. These stations in general do not correspond to the velocity data locations. Eight profiles are shown here: $x=-5.030, x=-3.009, x=-2.007, x=-1.007, x=-0.996, x=1.998, x=2.996$, and $x=6.173$ centimeters. These locations were selected to provide an even distribution of stations in the region of interest and be as close as possible to the velocity data presented above.

Density profiles are shown in Fig. 10. At the upstream location all models slightly over predict the density near the wall. Away from the wall the experiment shows a fuller density profile and the disagreement with the CFD solutions is more significant, resulting in the large over-predictions of displacement thickness shown earlier in Table 2. At the upstream location, where there is no shock interaction, the height of the thermal boundary layer can be inferred from the density profiles. The SA model produces the thickest thermal boundary layer. The $k-\epsilon$ model produces the thinnest thermal boundary layer and exhibits a sharper than 
expected curvature at the boundary layer edge. As one would expect from their similar formulation, the SST and BSL models show identical density profiles which lie in between the SST and $k-\epsilon$ solutions. In general, agreement in the interaction region is poor. The CFD solutions show the same general shape as the experiment, but misrepresent the size of the recirculation and magnitude of the density.

\section{Thermal Boundary Layer Effects}

The density data showed disagreement between the predicted and measured profiles at the upstream location. This disagreement affects the distribution of momentum in the boundary layer and may contribute to the large errors in the simulations in the shock-wave/boundary-layer interaction region. This section will describe two attempts to modify the thermal boundary layer, and hence the density and momentum in the boundary layer. Increasing the momentum near the wall, increases the flows ability to resist or delay separation. Reducing the momentum near the wall reduces the flow's ability to resist or delay separation. The intent is to gain some understanding of the impact on the overall prediction of the shock-wave/boundary-layer interaction.

\section{Effect of Turbulent Prandtl Number}

The standard approach in RANS analysis for computing the turbulent heat flux is to relate it to the turbulent momentum flux via the turbulent Prandtl number, $P r_{t}$. As was done in this study so far, the value of $P r_{t}$ is typically set to a constant value of 0.9 . However, it is known that the value can vary from approximately 0.5 to 2.0 in a boundary layer. ${ }^{19}$ Two additional cases were run using these two extreme values to ascertain the effect on the flow. The SA model was used because it showed the best agreement with the experimental data.

Figure 11 shows the velocity, temperature, and density profiles at the furthest upstream locations. Varying $\mathrm{Pr}_{t}$ had little visible effect on the velocity boundary layer, but altered the thickness and shape of the thermal boundary layer. Increasing $P r_{t}$ reduced the size of the thermal boundary layer, keeping the higher temperature flow closer to the wall. This reduced the density near the wall. Decreasing $\mathrm{Pr}_{t}$ has the opposite effect, increasing the size of the thermal boundary layer and diffusing the temperature more evenly through the boundary layer. This increased the density near the wall. Pressure distributions are shown in Fig. 12. The solution with the increased value of the turbulent Prandtl number showed an earlier pressure rise indicating the flow separated further upstream. The solution with the reduced value of $\operatorname{Pr}_{t}$ showed a more delayed separation. Skin friction plots (Fig. 13) confirm this finding and in addition show that there is a similar but smaller effect on the reattachment point. The $P r_{t}=2.0$ solution reattached further downstream than the baseline solution and the $\operatorname{Pr}_{t}=0.5$ solution reattached further upstream. Changing $\operatorname{Pr}_{t}$ also affected the skin friction in the upstream portion of the boundary layer. The data are summarized in Table 4 and show that the $P r_{t}=2.0$ solution increased the separation length by 30 percent and the $P r_{t}=0.5$ solution reduced the separation length by 18 percent.

Table 4: Effect of turbulent Prandtl number on the separated region

\begin{tabular}{|c|c|c|c|c|c|}
\hline Model & Wall Temp. (K) & $P r_{t}$ & $\begin{array}{c}\text { Separation } \\
\text { Location }(\mathrm{cm})\end{array}$ & $\begin{array}{l}\text { Reattachment } \\
\text { Location }(\mathrm{cm})\end{array}$ & $\begin{array}{c}\text { Separation } \\
\text { Length }(\mathrm{cm}) \\
\end{array}$ \\
\hline Experiment (oil flow) & & & -2.73 & 0.97 & 3.70 \\
\hline SA & adiabatic & 0.9 & -2.6750 & 2.2736 & 4.9236 \\
\hline SA & adiabatic & 0.5 & -2.0754 & 1.9656 & 4.0410 \\
\hline SA & adiabatic & 2.0 & -3.6875 & 2.7090 & 6.3965 \\
\hline
\end{tabular}

It was shown that the turbulent Prandtl number can have a significant effect. Setting $P r_{t}=2.0$ improved the prediction of the density profile near the boundary layer edge, but worsened the prediction near the wall. Kays showed that the turbulent Prandtl number varies through the boundary layer, from 0.5 near the wall to 2.0 at the boundary layer edge. ${ }^{19}$ The ability to vary the $P r_{t}$ through the boundary layer may improve the current predictions of the density profile. There are several variable $P r_{t}$ models that could be applied to this problem and may improve the prediction. ${ }^{20-22}$ This should be the subject of further investigation. 


\section{Effect of Wall Temperature}

The effect of the wall temperature is examined next. For the previous discussions in this paper, all the simulations used an adiabatic condition on the solid walls. The simulations indicated that the wall temperature at the $x=-4.500 \mathrm{~cm}$ location resulting from the adiabatic assumption was approximately $246 \mathrm{~K}$ for all turbulence models, yielding a recovery factor of 0.86. A cooler wall would increase the density and momentum there, increasing the flow's ability to overcome or delay separation, and a hotter wall would have the opposite effect. Two additional cases were run using the SA model, where the wall temperature was set to a constant value. The temperatures selected were: 1) $170 \mathrm{~K}$, and 2) $270 \mathrm{~K}$, the freestream stagnation temperature. Subsequent to running these analyses, we became aware of the work of Wideman, who conducted wall temperature measurements to support his LISF work on a similar geometry. ${ }^{9}$ His data was limited to one measurement located on the flare of the model. It showed that the wall temperature tracked the freestream stagnation temperature very closely.

Upstream profiles of velocity and density are shown in Fig. 14. There is no noticeable effect on the velocity profile. The density profiles show the increased density near the wall for the cold wall case. The hot wall case, $T_{w}=270 \mathrm{~K}$, shows only a slightly larger thermal boundary layer. Pressure distributions (Fig. 15) and skin friction distributions (Fig. 16) show that a cold wall can significantly delay the separation point, reduce the overall separation length, and increase the extent and magnitude of the primary pressure rise. The upstream skin friction coefficient remains within the experiment measurements for the hot wall case and the separation point moved upstream, closer to the experimental value. The separation and reattachment locations are tabulated in Table 5. While the above evidence suggests that setting the wall temperature

Table 5: Effect of wall temperature on the separated region

\begin{tabular}{|c|c|c|c|c|c|}
\hline Model & Wall Temp. (K) & $P r_{t}$ & $\begin{array}{c}\text { Separation } \\
\text { Location }(\mathrm{cm})\end{array}$ & $\begin{array}{l}\text { Reattachment } \\
\text { Location }(\mathrm{cm})\end{array}$ & $\begin{array}{c}\text { Separation } \\
\text { Length }(\mathrm{cm})\end{array}$ \\
\hline Experiment (oil flow) & & & -2.73 & 0.97 & 3.70 \\
\hline SA & adiabatic & 0.9 & -2.6750 & 2.2736 & 4.9236 \\
\hline SA & 170.0 & 0.9 & -1.9266 & 1.7767 & 3.7033 \\
\hline SA & 270.0 & 0.9 & -2.9125 & 2.4227 & 5.3352 \\
\hline
\end{tabular}

to the tunnel total temperature would improve the comparisons with the experiment, the velocity, shear stress, and density profiles did not substantiate this assertion. Although the plots are not shown for brevity, the profiles from the $T_{w}=270 \mathrm{~K}$ case were uniformly similar to the adiabatic profiles, but agreement with experiment was slightly worse.

\section{Conclusions}

A CFD study of a turbulent shock-wave/boundary-layer interaction created by an axisymmetric compression corner was carried out to assess its suitability as a standard test case for turbulence model development and assessment. The case was selected because its simple geometry reduces the complexity of the analysis, but still retains the key flow features that will challenge RANS turbulence models.

The flowfield was shown to contain a complex interaction of a primary oblique shock wave, large boundary layer separation, separation induced shock, and third shock behind the separation shock wave, which coalesces with the primary shock wave. A large expansion wave turns the flow back axially, downstream of the compression flare. A small counter-rotating vortex sits between the compression corner and the separated region.

Four different turbulence models were compared with experimental data: Spalart-Allmaras, Menter BSL, Menter SST, and $k-\epsilon$ AKN. An experimental separation point was estimated using the oil flow visualization and surface pressure distribution. It was found that a very fine grid of over 900,000 points was necessary to achieve grid independence. The SST model predicted the largest separated region, with separation point upstream of the experimental result. The other three models predicted the separation point downstream of the experimental value, with the SA model being closest to the experimental estimate. The SA model also showed the best agreement with the experimental pressure distribution. 
Velocity profiles indicated that all models appear to predict a stronger recirculation in the separated region. Shear stress profiles were poorly predicted for all turbulence models.

Density profiles indicate that the thermal boundary layer is not correctly predicted prior to the interaction region. The effect of turbulent Prandtl number and wall temperature were studied in an attempt to better match the experimental data and study the effects on the interaction region. Altering the constant turbulent Prandtl number did not improve the agreement with the upstream flow profiles, but did have a significant effect on the separation and reattachment locations. While agreement with the experimental data was not improved, the data suggests that a variable Prandtl number model may be beneficial. Two additional wall temperature cases were studied: $170 \mathrm{~K}$ and $270 \mathrm{~K}$. The colder wall temperature delayed separation and speeded reattachment. The hotter wall temperature moved the separation upstream, closer to the experimental data and delayed reattachment. While there is some limited experimental evidence that suggests this is the correct wall temperature, it did not improve the agreement with the experimental flow profiles in the interaction region.

This relatively simple shock-wave/boundary-layer interaction problem proved to be very challenging to predict. It appears to be a good candidate for use in measuring future progress in turbulence model development because of its challenging nature, easy to analyze geometry, and complex flow physics.

\section{Acknowledgements}

This work was sponsored by the National Aeronautics and Space Administration's Aeronautical Sciences Project, Revolutionary Computational Aerosciences Sub-project. The author would like to thank Dr. James L. Brown, Dr. Dennis Yoder, Dr. Nicholas Georgiadis, and the AIAA Turbulence Model Benchmarking Working Group for their advice and insights.

\section{References}

\footnotetext{
${ }^{1}$ Choi, H. and Moin, P., "Grid-point requirements for large eddy simulation: Chapman's estimates revisited," Physics of Fluids, Vol. 24, No. 1, 2012, pp. 011702-011702-5.

${ }^{2}$ Slotnick, J., Khodadoust, A., Alonso, J., Darmofal, D., Gropp, W., Lurie, E., and Mavriplis, D., "CFD Vision 2030 Study: A Path to Revolutionary Computational Aerosciences," NASA CR 2014-218178, 2014.

${ }^{3}$ Delery, J. and Marvin, J. G., "Shock-Wave Boundary Layer Interactions," AGARDograph No. 280, 1986, E. Reshotko (ed.).

${ }^{4}$ Benek, J. and Babinsky, H., "Lessons Learned from the 2010 AIAA Shock Boundary Layer Interaction Workshop," AIAA Paper 2010-4825, 2010.

${ }^{5}$ DeBonis, J. R., Oberkampf, W. L., Wolf, R. T., Orkwis, P. D., and Turner, M. G., "Assessment of Computational Fluid Dynamics and Experimental Data for Shock Boundary-Layer Interactions," AIAA Journal, Vol. 50, No. 4, 2012 , pp. 891-903.

${ }^{6}$ Dunagan, S. E., Brown, J. L., and Miles, J. B., "Interferometric Data for a Shock-Wave/Boundary-Layer Interaction," NASA TM 88227, 1986.

${ }^{7}$ Brown, J., Kussoy, M., and Coakley, T., "Turbulent Properties of Axisymmetric Shock-Wave/Boundary-Layer Interaction Flows," Turbulent Shear-Layer/Shock-Wave Interactions, edited by J. Delery, Springer-Verlag, 1986, pp. 137-148.

${ }^{8}$ Brown, J. D., Brown, J. L., and Kussoy, M. I., "A Documentation of Two- and Three-Dimensional Shock-Separated Turbulent Boundary Layers," NASA TM 101008, 1988.

${ }^{9}$ Wideman, J., Brown, J., Miles, J., and Ozcan, O., "Surface Documentation of a 3-D Supersonic, Shock-Wave/BoundaryLayer Interaction," NASA TM 108824, 1994.

${ }^{10}$ Settles, G. S. and Dodson, L. J., "Hypersonic Shock/Boundary-Layer Interaction Database," NASA CR $177577,1991$.

${ }^{11}$ Brown, J. L., personal communication.

${ }^{12}$ Pointwise, Inc., http://www.pointwise.com/gridgen/.

${ }^{13}$ The NPARC Alliance, http://www.grc.nasa.gov/www/winddocs/.

${ }^{14}$ Kandula, M. and Buning, P., "Implementation of LU-SGS Algorithm and Roe Upwinding Scheme in OVERFLOW Thin-Layer Navier-Stokes Code," AIAA Paper 94-2357, 1994.

${ }^{15}$ Spalart, P. R. and Allmaras, S. R., "A One-Equation Turbulence Model for Aerodynamic Flows," La Recherche Aerospatiale, No. 1, 1994, pp. 5-21.

${ }^{16}$ Menter, F. R., "Two-Equation Eddy-Viscosity Turbulence Models for Engineering Applications," AIAA Journal, Vol. 32, No. 8, 1994, pp. 1598-1605.

${ }^{17}$ Abe, K., Kondoh, T., and Nagano, Y., "A New Turbulence Model for Predicting Fluid Flow and Heat Transfer in Separating and Reattaching Flows-I. Flow Field Calculations," International Journal of Heat and Mass Transfer, Vol. 37, No. 1, 1994, pp. 139-151.

${ }^{18}$ Chien, K.-Y., "Predictions of Channel and Boundary Layer Flows with a Low-Reynolds-Number Turbulence Model," AIAA Journal, Vol. 20, No. 1, 1982, pp. 33-38.

${ }^{19}$ Kays, W. M., "Turbulent Prandtl Number - Where Are We?" Transactions of the ASME, Journal of Heat Transfer, Vol. 116, May 1994, pp. 284-295.
} 
${ }^{20}$ Nagano, Y. and Kim, C., "A Two-Equation Model for Heat Transport in Wall Turbulent Shear Flows," Journal of Heat Transfer, Vol. 110, Aug. 1988, pp. 583-589.

${ }^{21}$ Nagano, Y., Hattori, H., and Abe, K., "Modeling the Turbulent Heat and Momentum Transfer in Flows Under Different Thermal Conditions," Fluid Dynamics Research, Vol. 20, 1997, pp. 127-142.

${ }^{22}$ Sommer, T., So, R., and Zhang, H., "Supersonic Flow Calculations Using a Reynolds-Stress and a Thermal Eddy Diffusivity Turbulence Model," Journal of Fluids Engineering, Vol. 116, Sept. 1994, pp. 469-476.

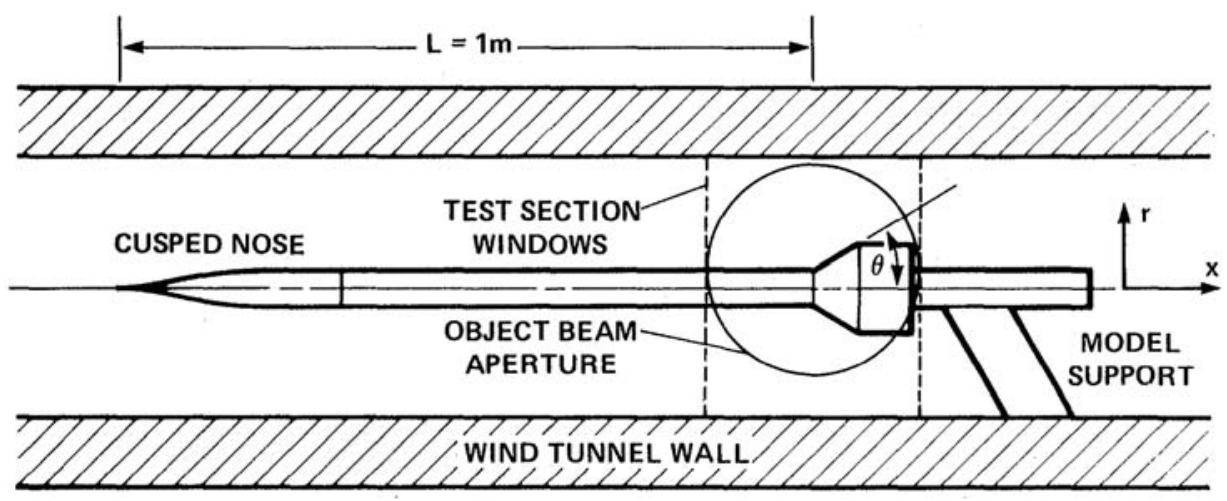

Figure 1: Experimental configuration, from Dunagan et al. ${ }^{6}$ 


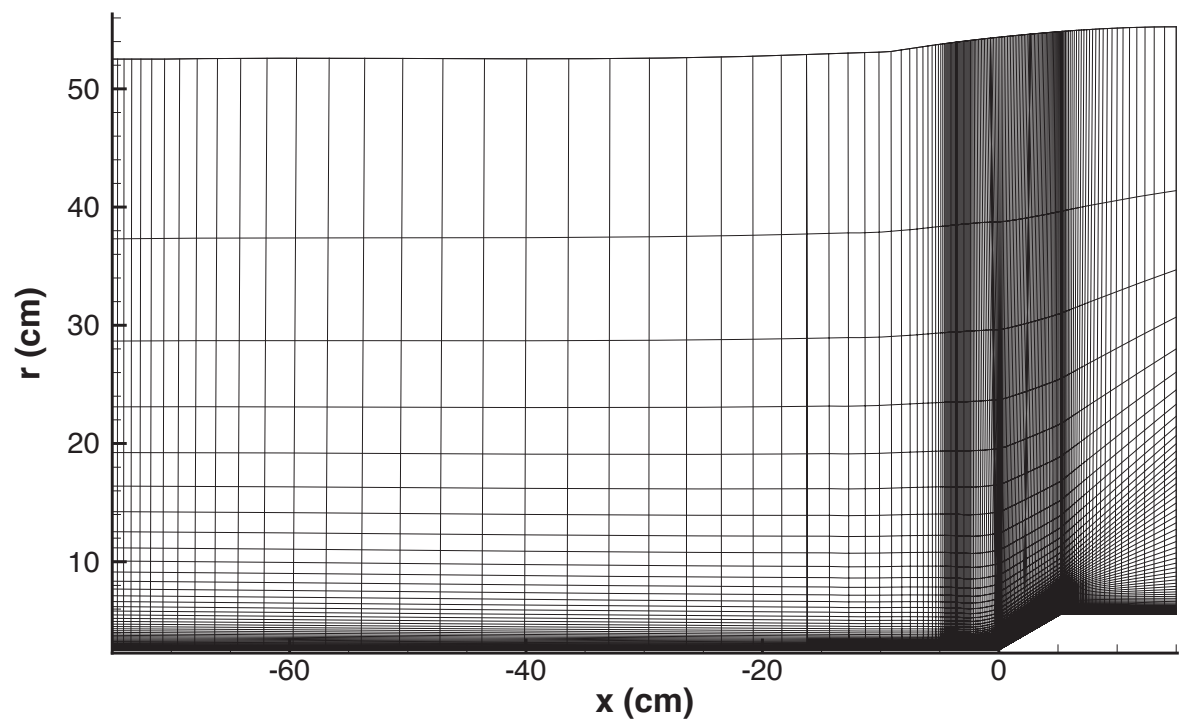

(a) Complete domain

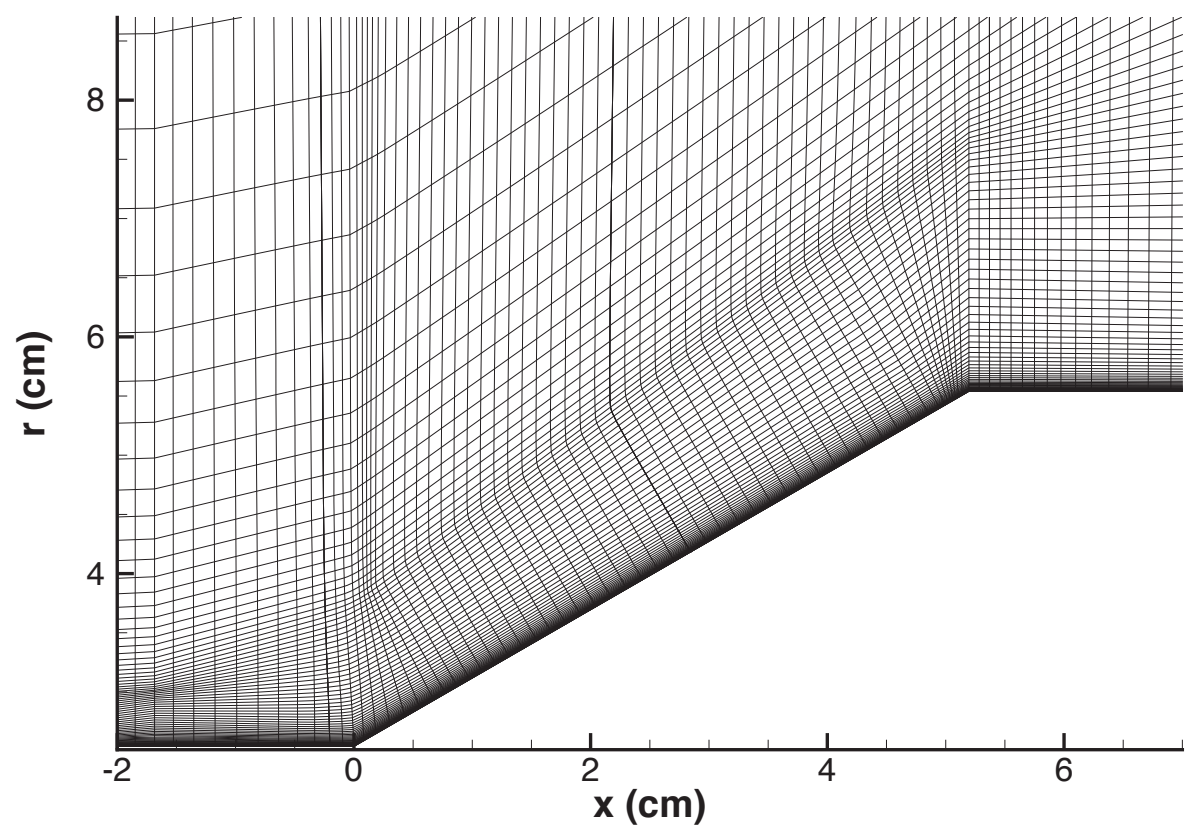

(b) Close-up of flare

Figure 2: Computational grid, every 8th grid point shown 


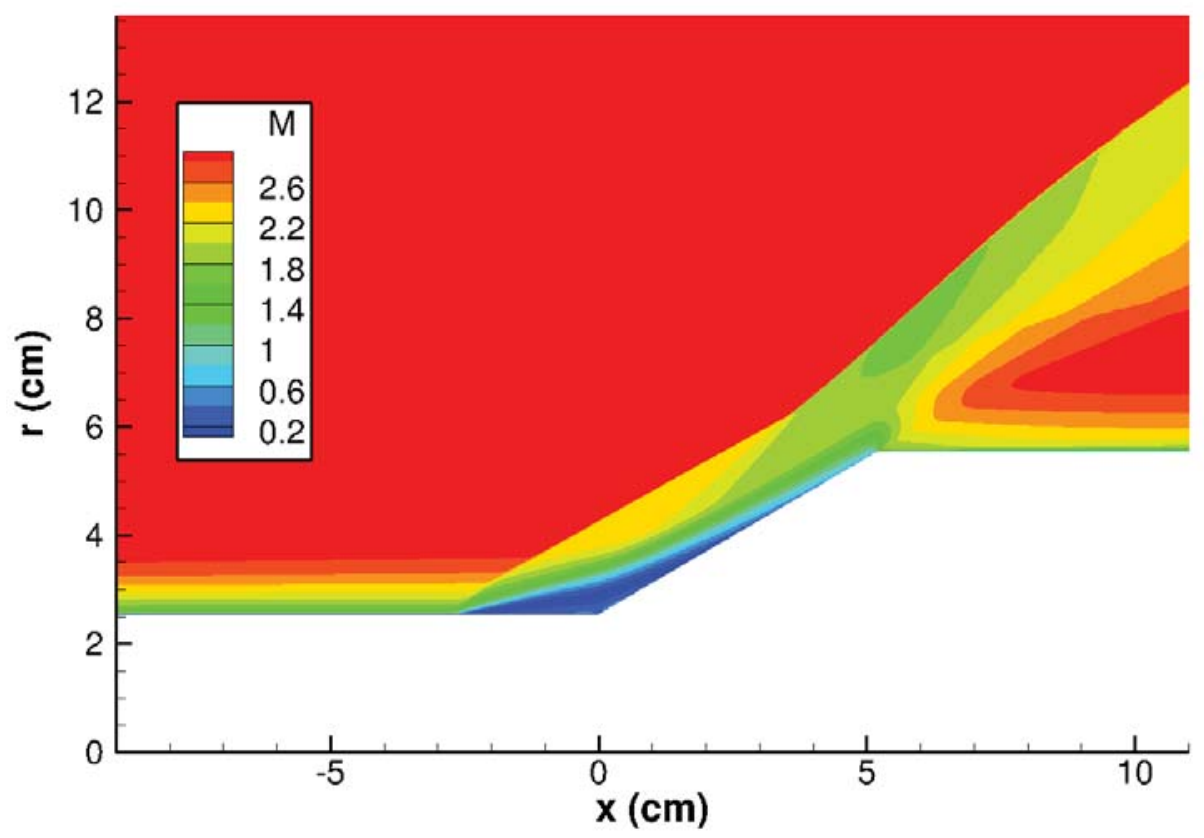

(a) Mach number contours

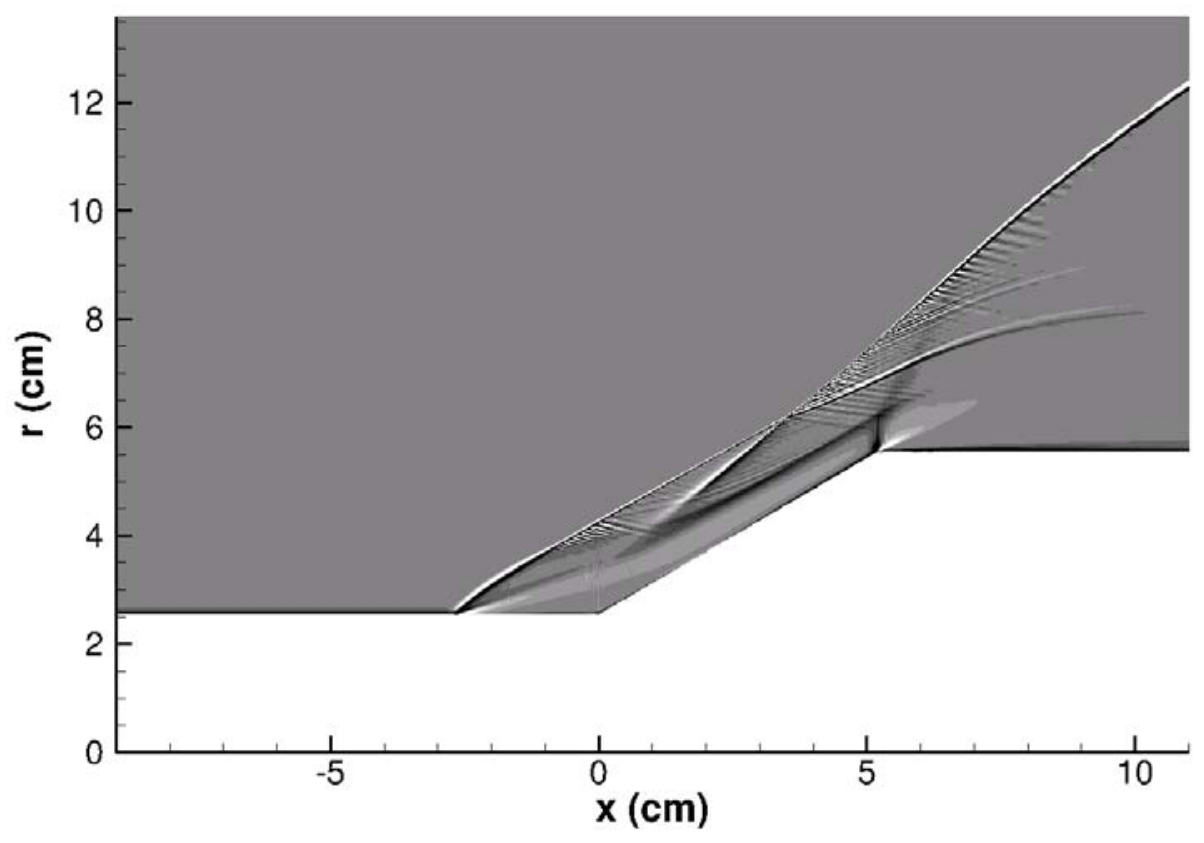

(b) Numerical shadowgraph

Figure 3: Depiction of the shock-wave/boundary-layer interaction region 


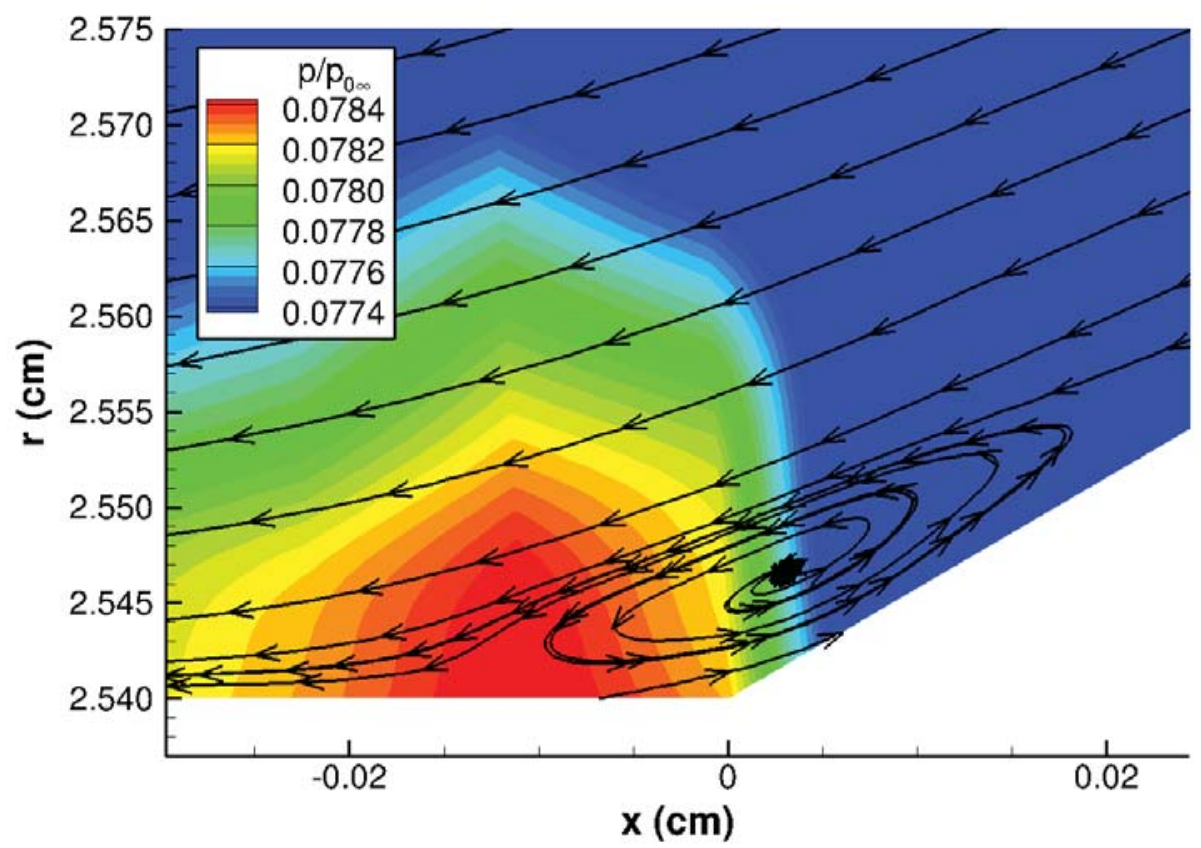

Figure 4: Streamlines and pressure contours in corner region

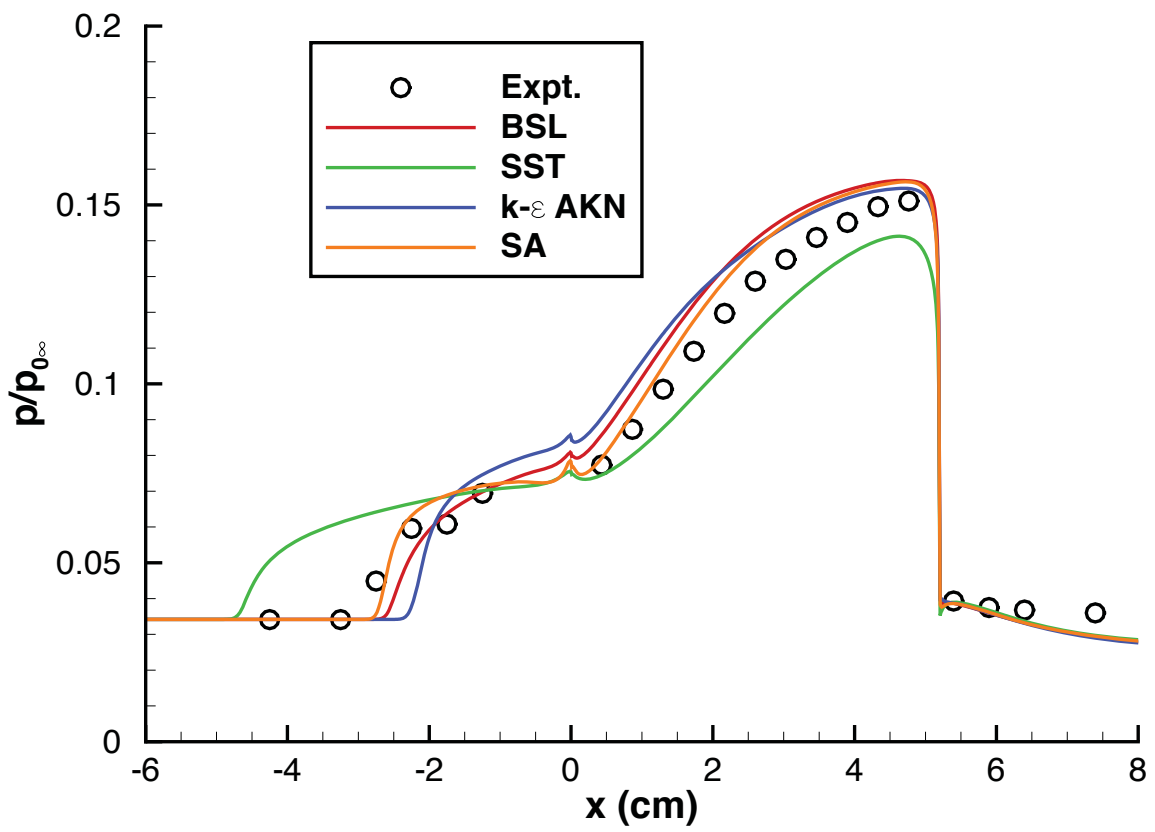

Figure 5: Turbulence model comparison for surface static pressure coefficient, $p / p_{0 \infty}$ 


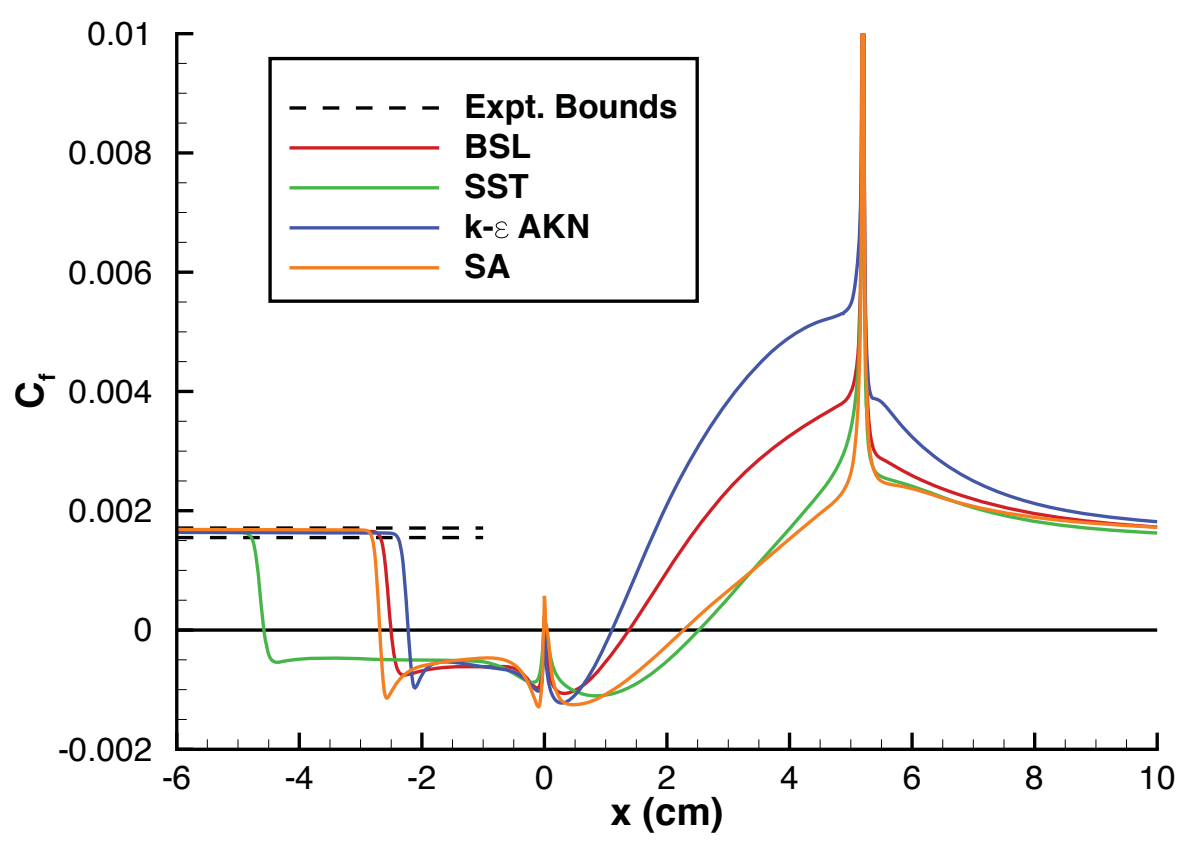

Figure 6: Turbulence model comparison for skin friction coefficient, $C_{f}$ 


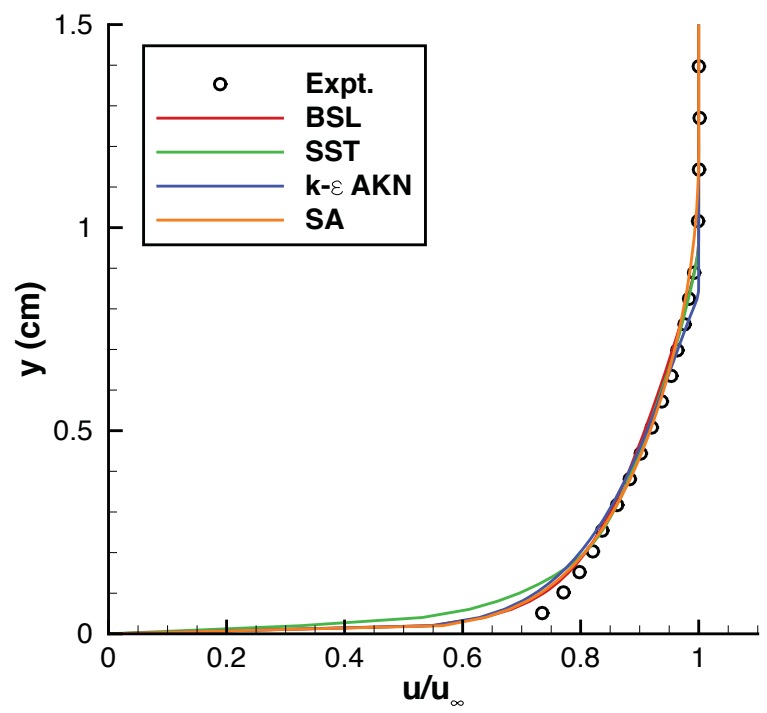

(a) $x=-4.500 \mathrm{~cm}$

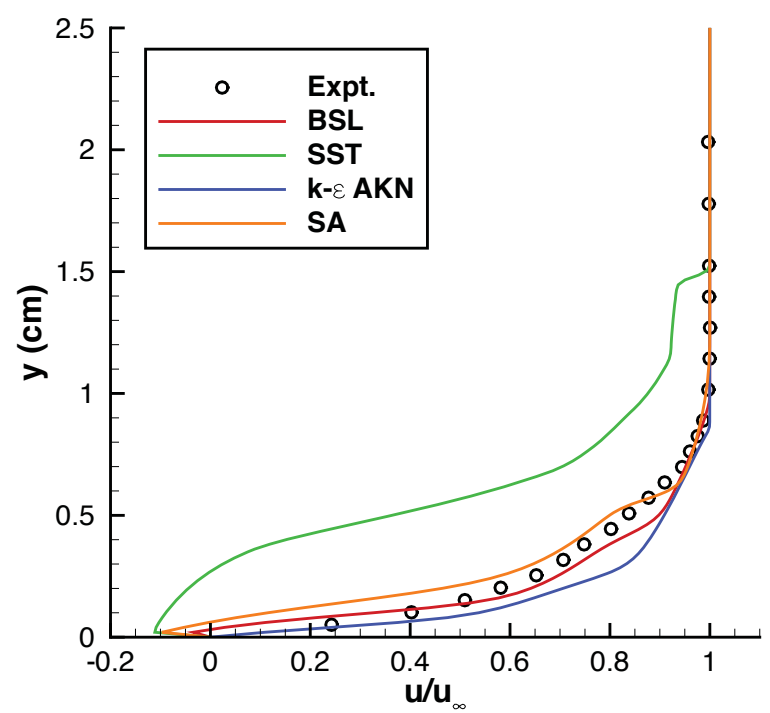

(c) $x=-2.000 \mathrm{~cm}$

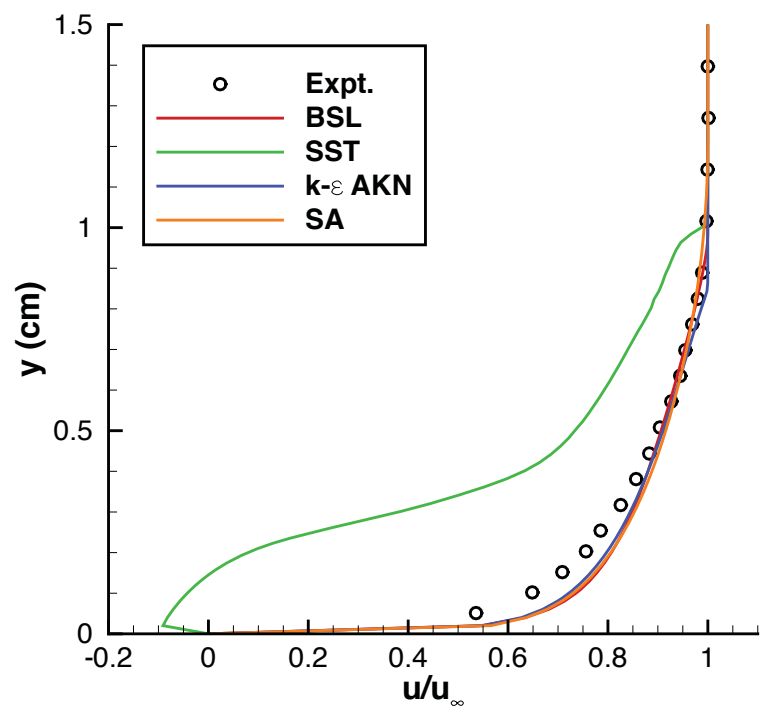

(b) $x=-3.000 \mathrm{~cm}$

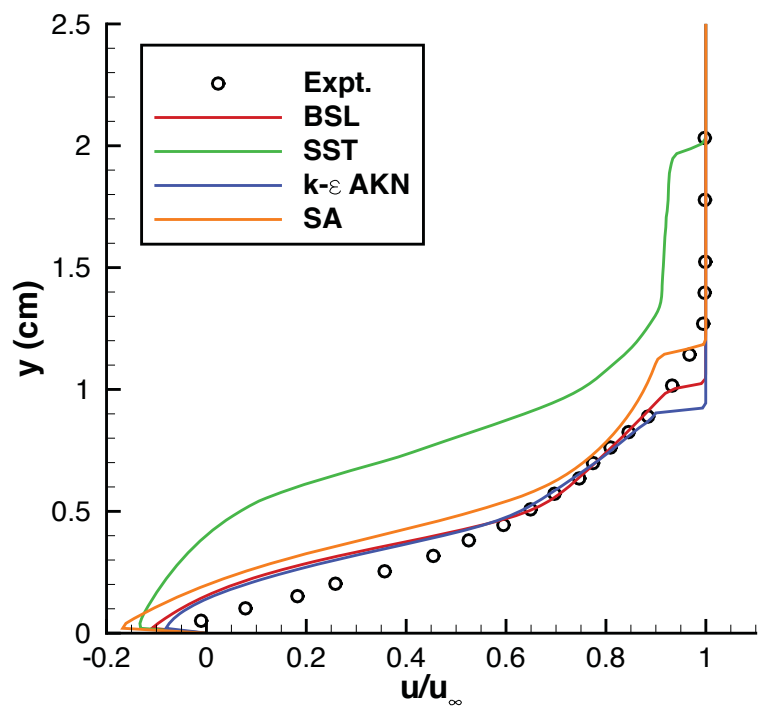

(d) $x=-1.000 \mathrm{~cm}$

Figure 7: Turbulence model comparison for axial velocity profiles, $u / u_{\infty}$ 


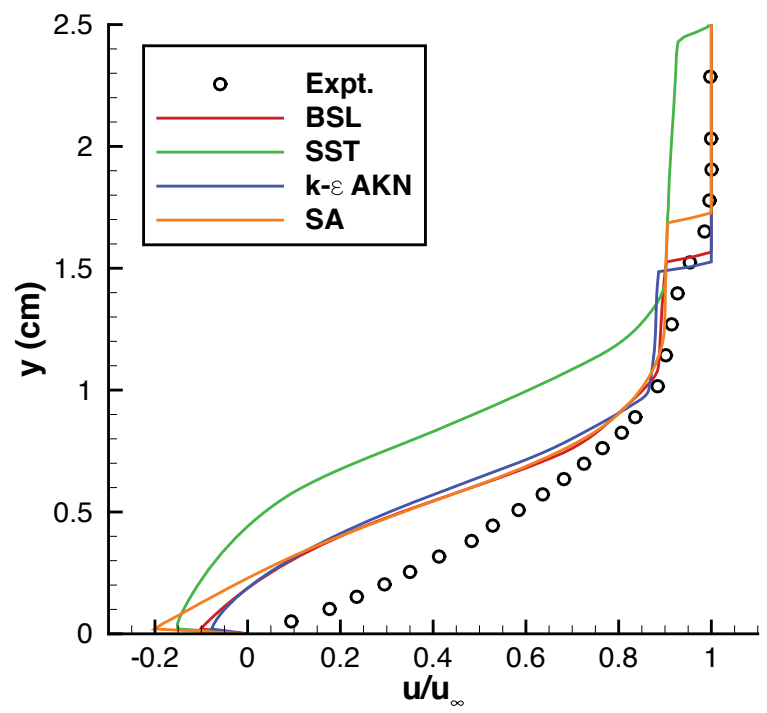

(e) $x=0.433 \mathrm{~cm}$

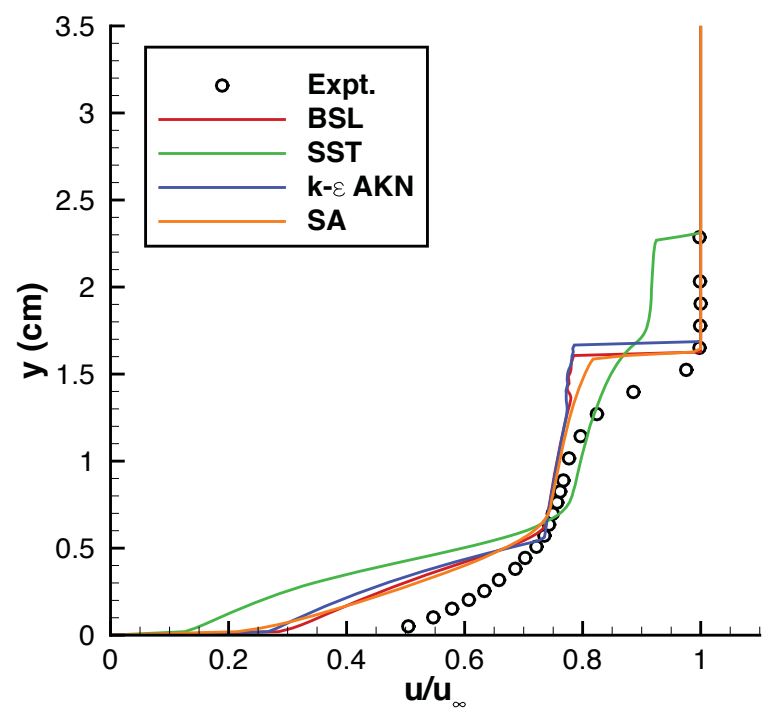

(g) $x=3.464 \mathrm{~cm}$

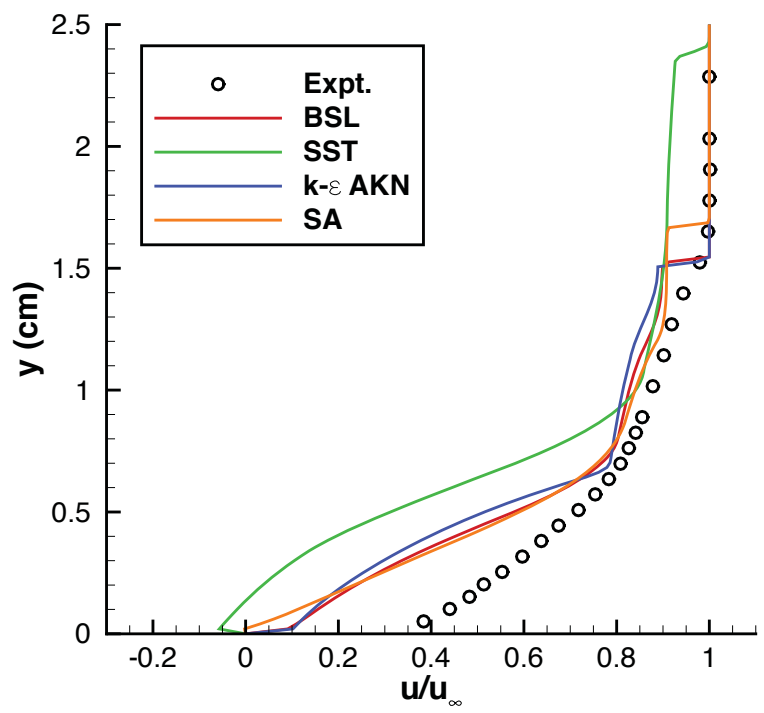

(f) $x=1.732 \mathrm{~cm}$

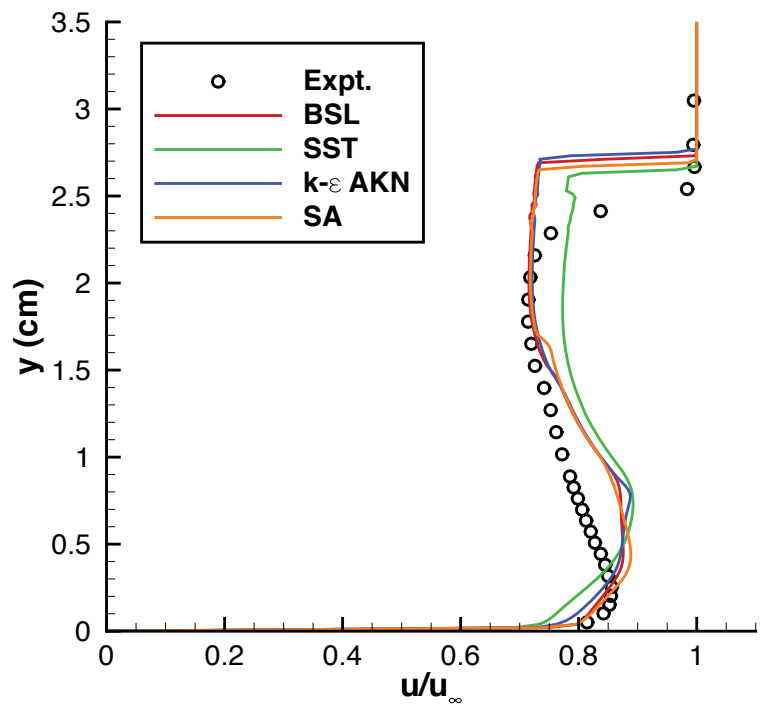

(h) $x=5.896 \mathrm{~cm}$

Figure 7: (continued) Turbulence model comparison for axial velocity profiles, $u / u_{\infty}$ 


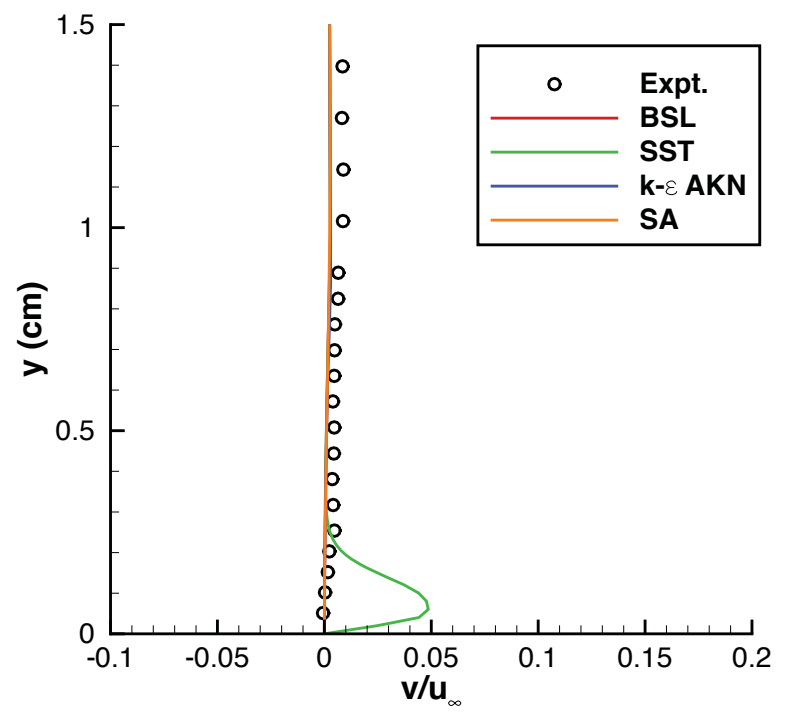

(a) $x=-4.500 \mathrm{~cm}$

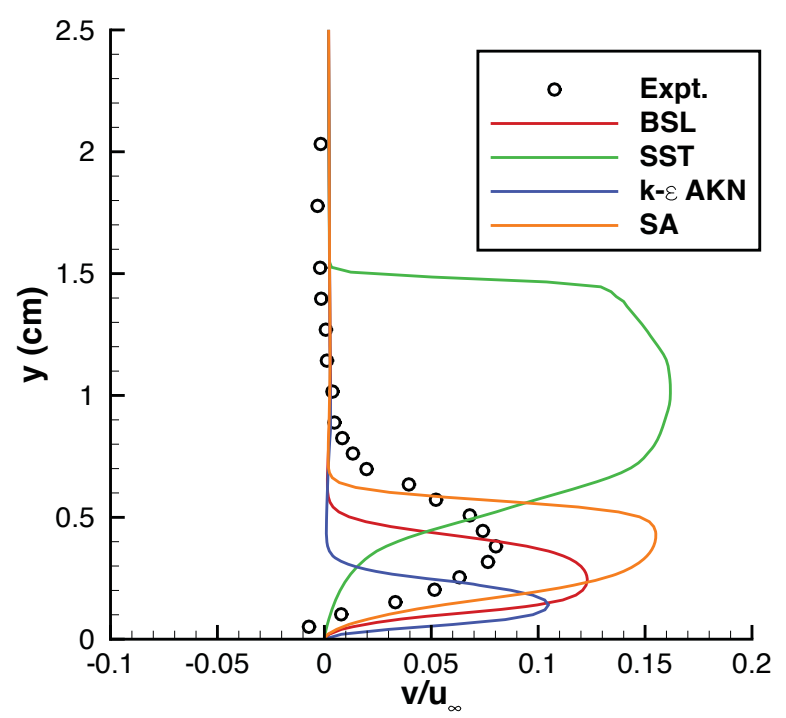

(c) $x=-2.000 \mathrm{~cm}$

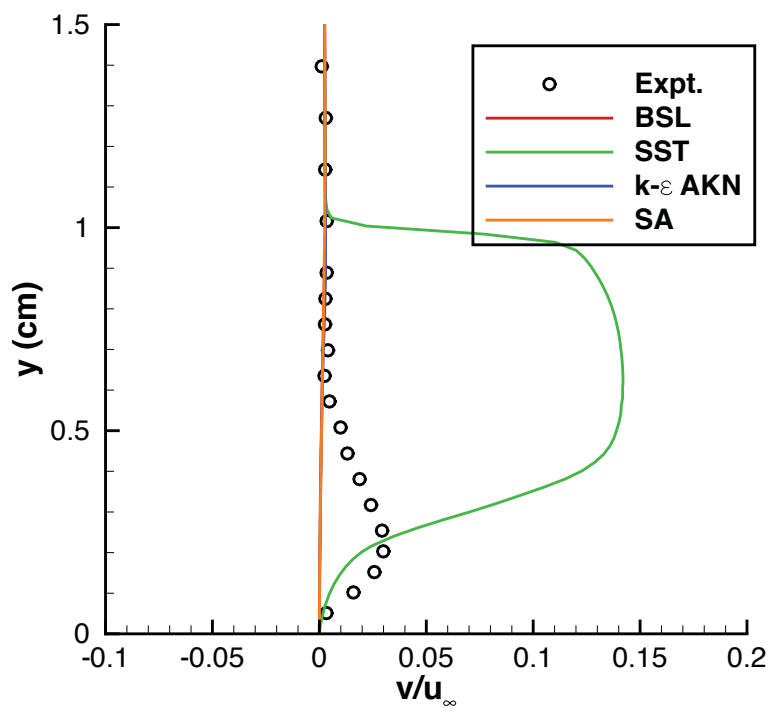

(b) $x=-3.000 \mathrm{~cm}$

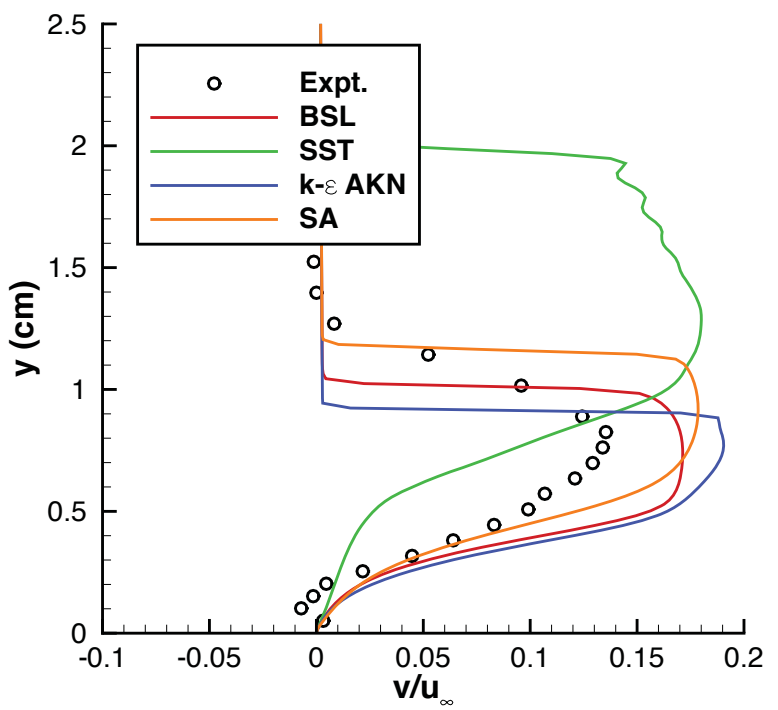

(d) $x=-1.000 \mathrm{~cm}$

Figure 8: Turbulence model comparison for radial velocity profiles, $v / u_{\infty}$ 


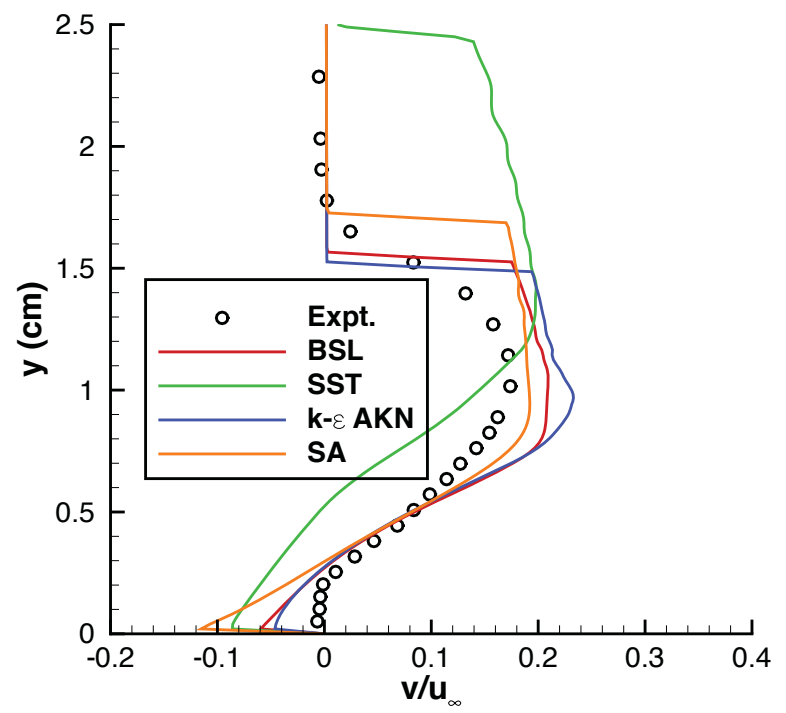

(e) $x=0.433 \mathrm{~cm}$

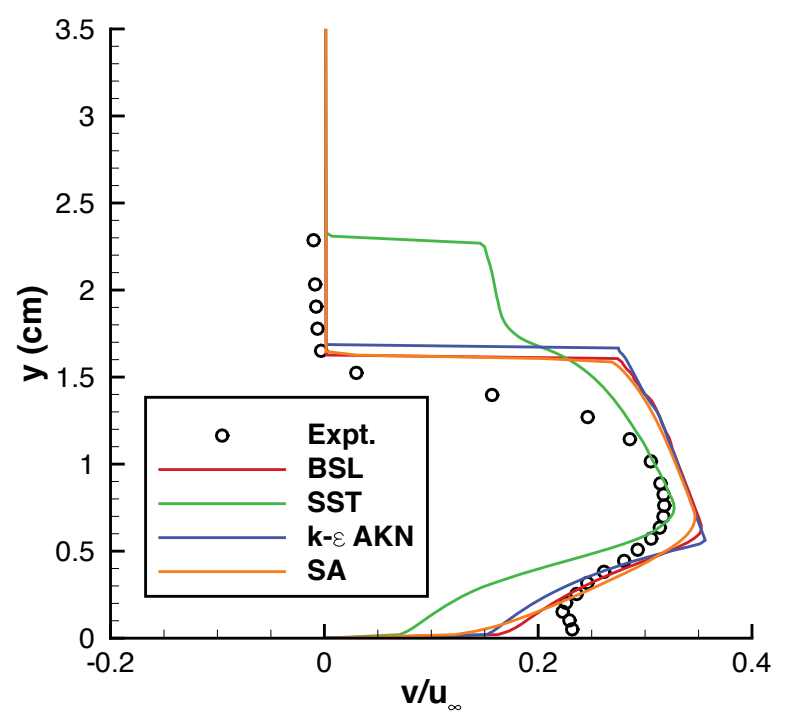

(g) $x=3.464 \mathrm{~cm}$

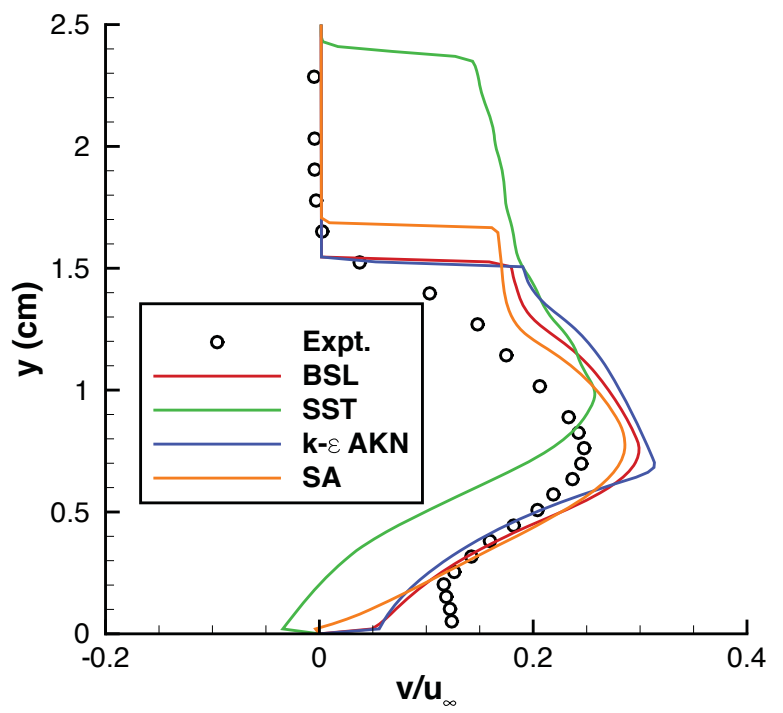

(f) $x=1.732 \mathrm{~cm}$

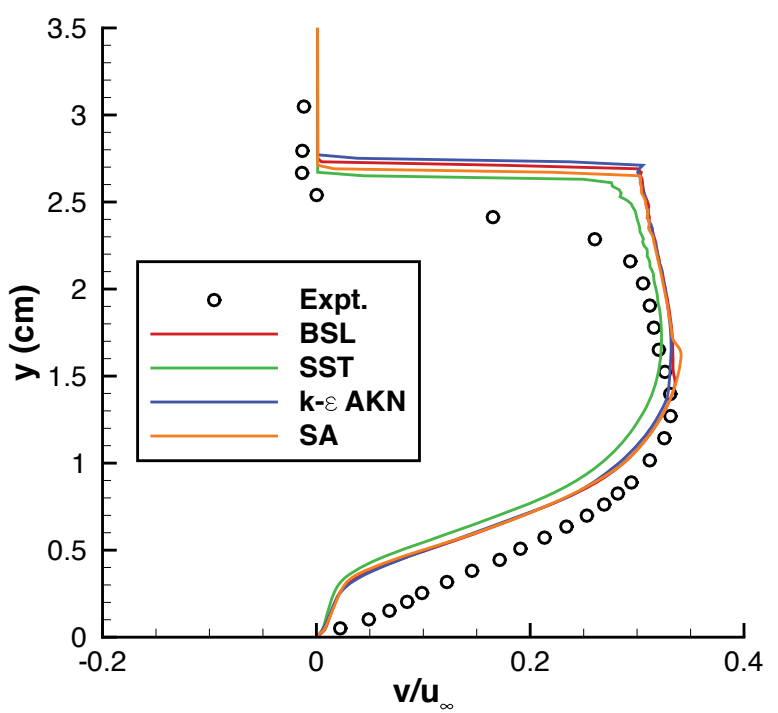

(h) $x=5.896 \mathrm{~cm}$

Figure 8: (continued) Turbulence model comparison for radial velocity profiles, $v / u_{\infty}$ 


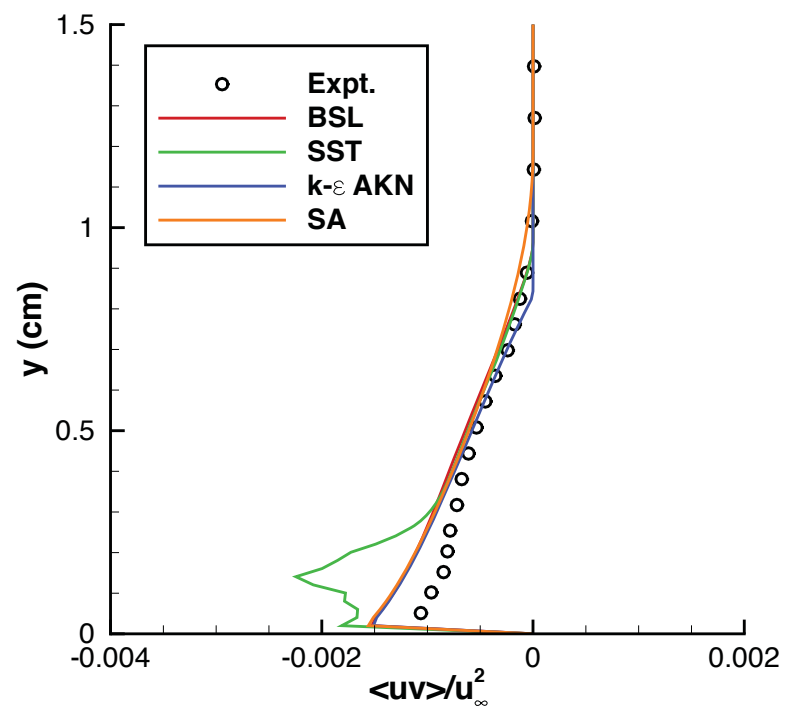

(a) $x=-4.500 \mathrm{~cm}$

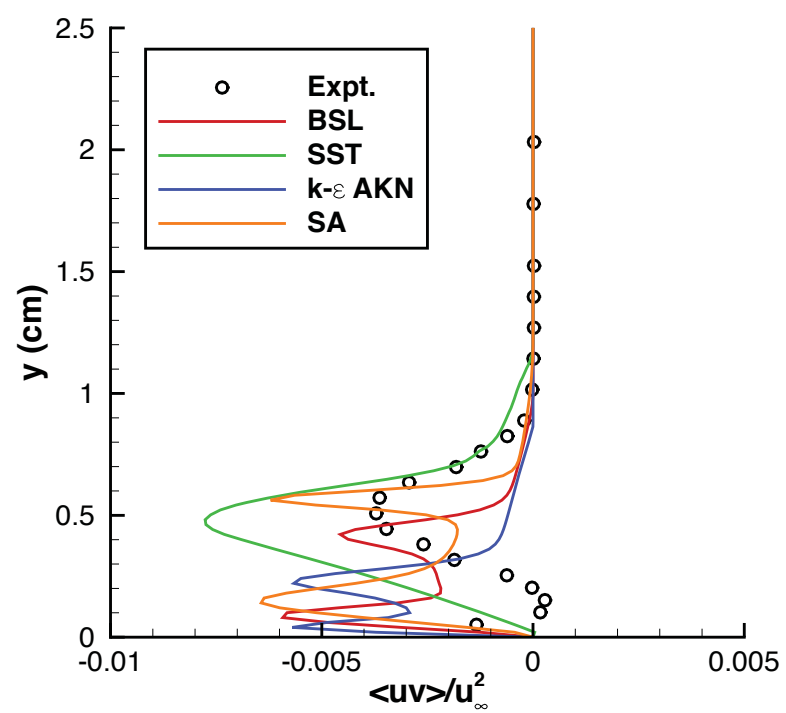

(c) $x=-2.000 \mathrm{~cm}$

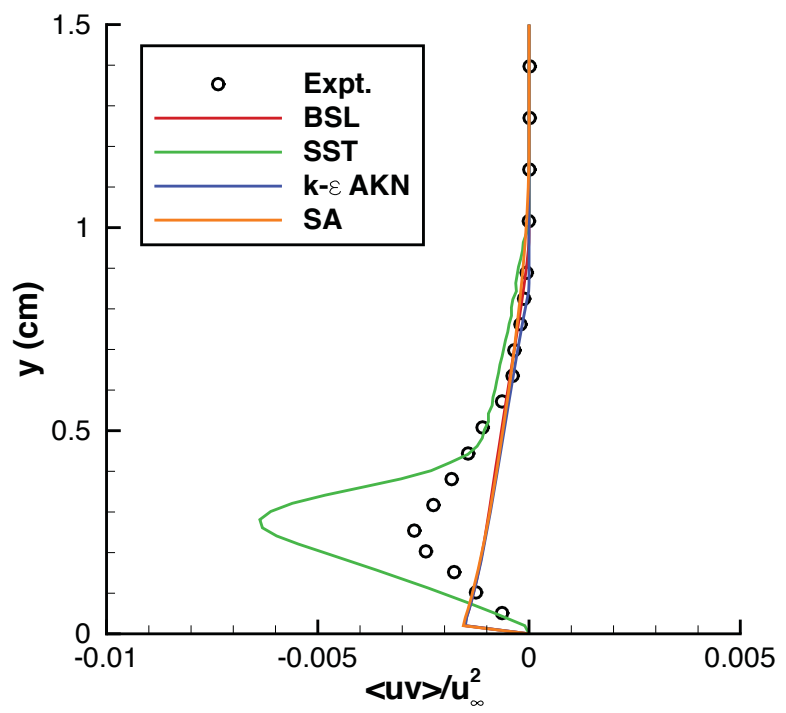

(b) $x=-3.000 \mathrm{~cm}$

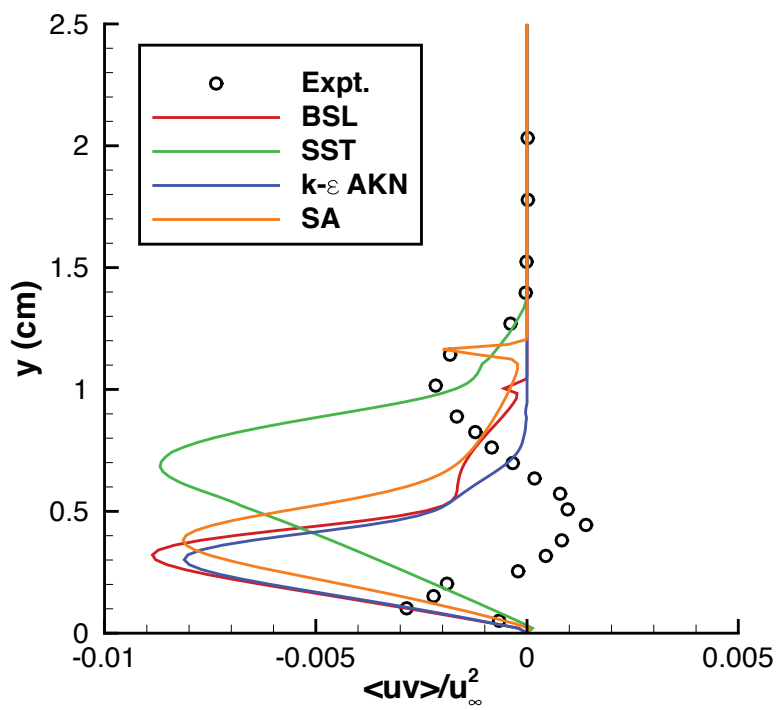

(d) $x=-1.000 \mathrm{~cm}$

Figure 9: Turbulence model comparison for shear stress profiles, $\langle u v\rangle / u_{\infty}^{2}$ 


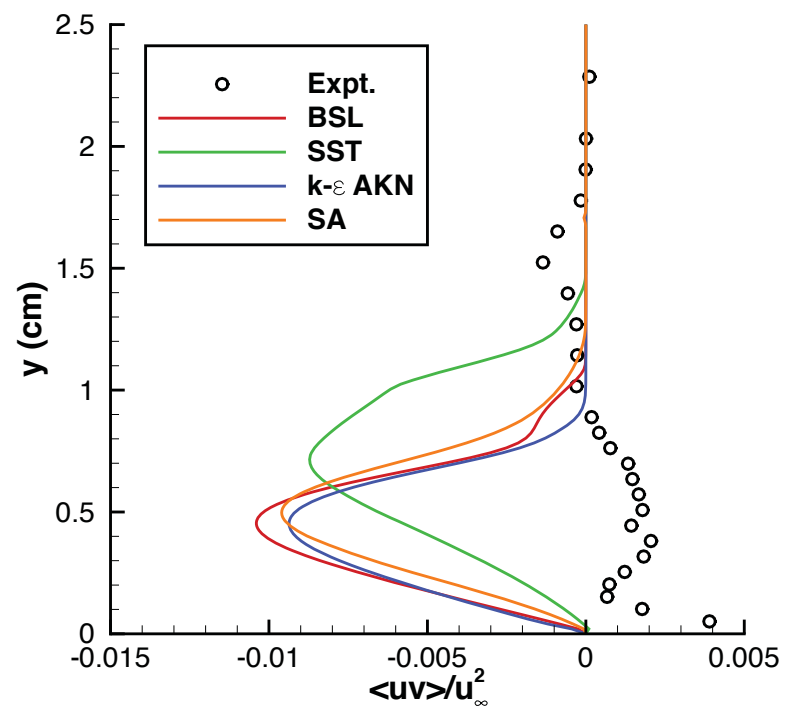

(e) $x=0.433 \mathrm{~cm}$

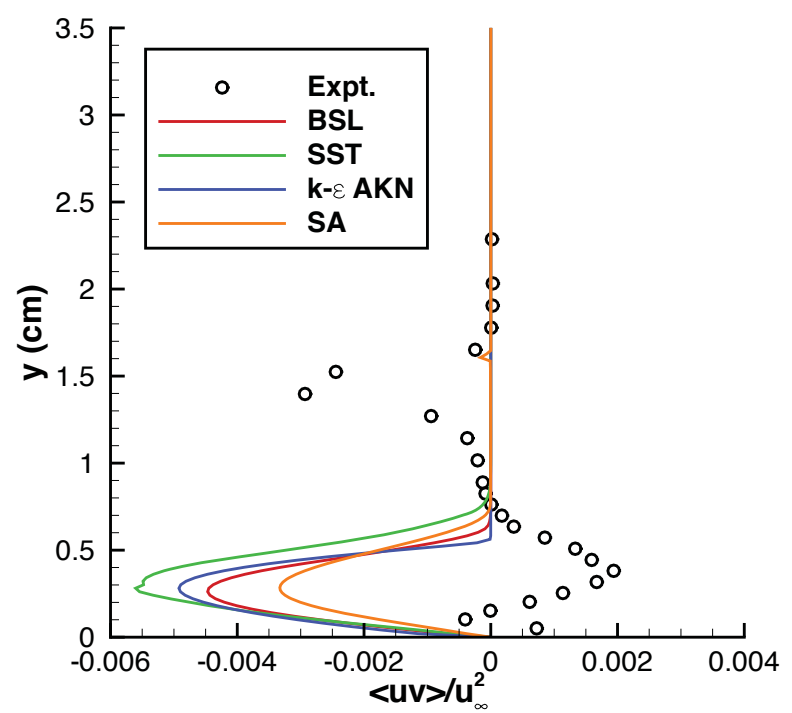

(g) $x=3.464 \mathrm{~cm}$

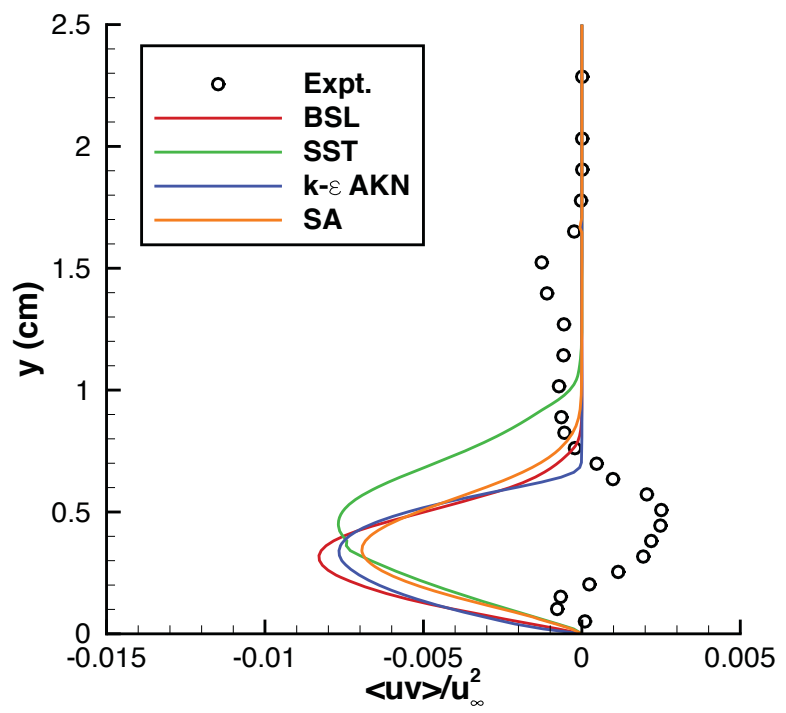

(f) $x=1.732 \mathrm{~cm}$

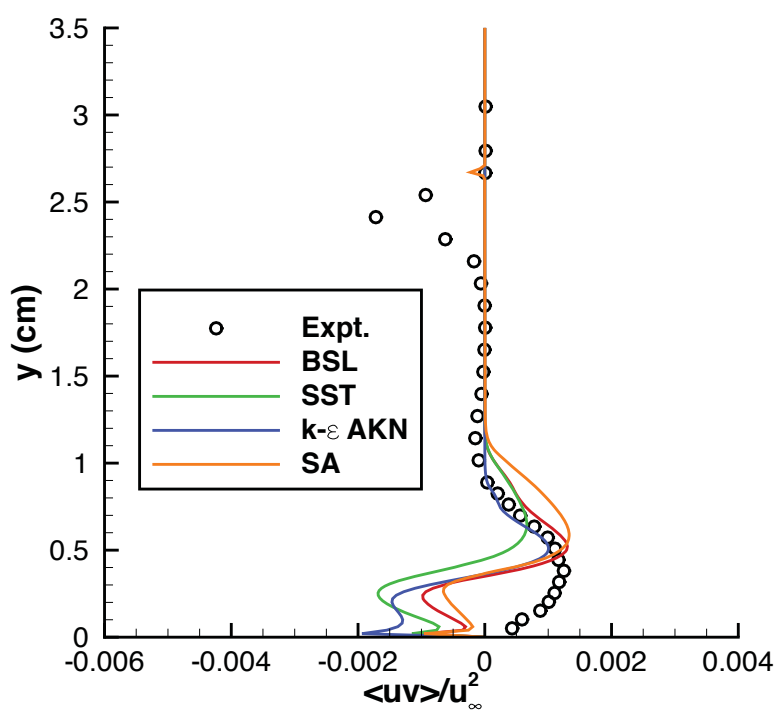

(h) $x=5.896 \mathrm{~cm}$

Figure 9: (continued) Turbulence model comparison for shear stress profiles, $\langle u v\rangle / u_{\infty}^{2}$ 


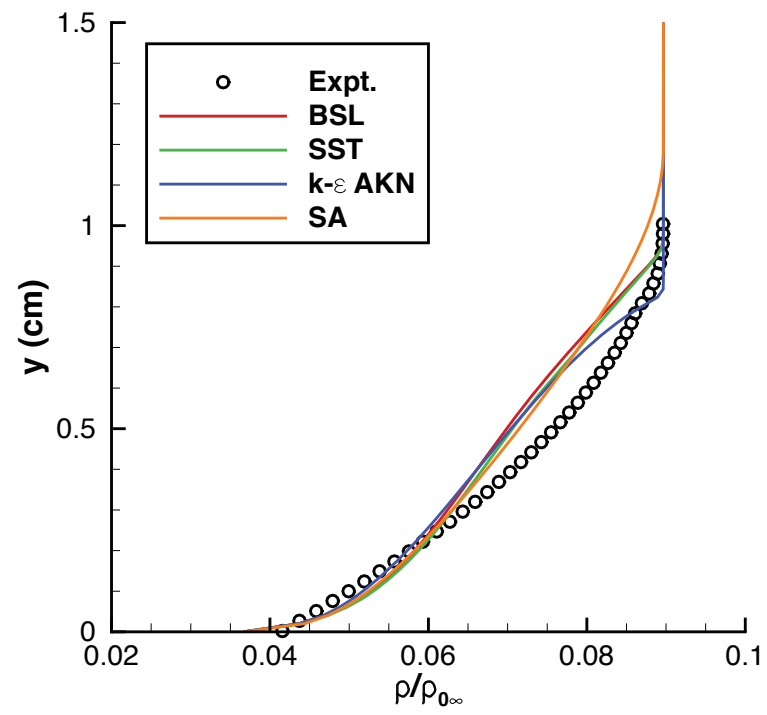

(a) $x=-5.030 \mathrm{~cm}$

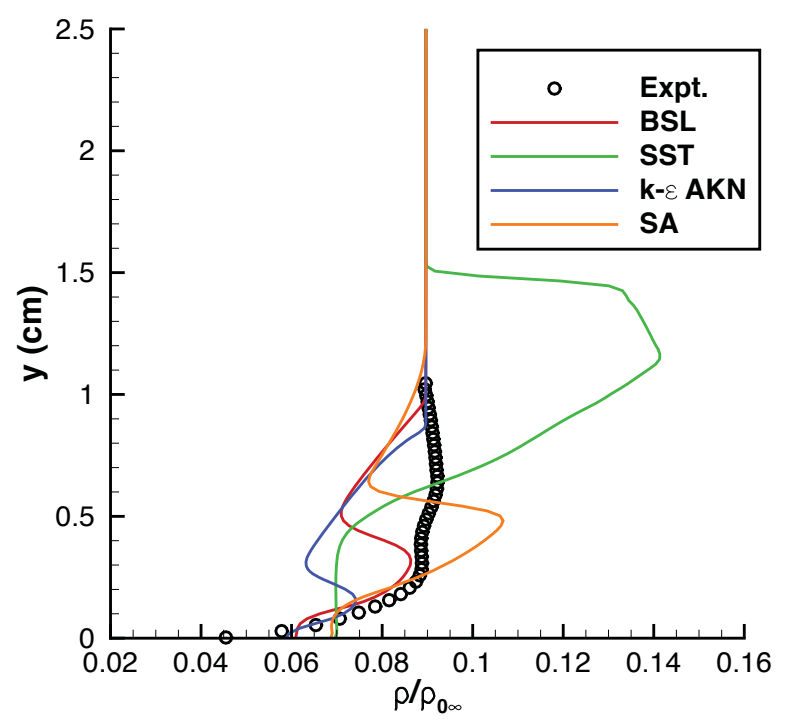

(c) $x=-2.007 \mathrm{~cm}$

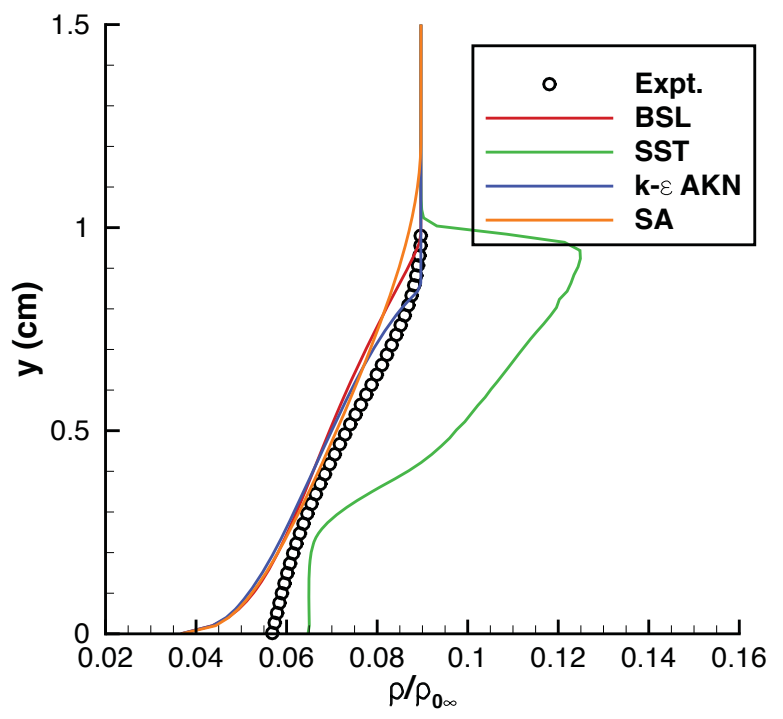

(b) $x=-3.009 \mathrm{~cm}$

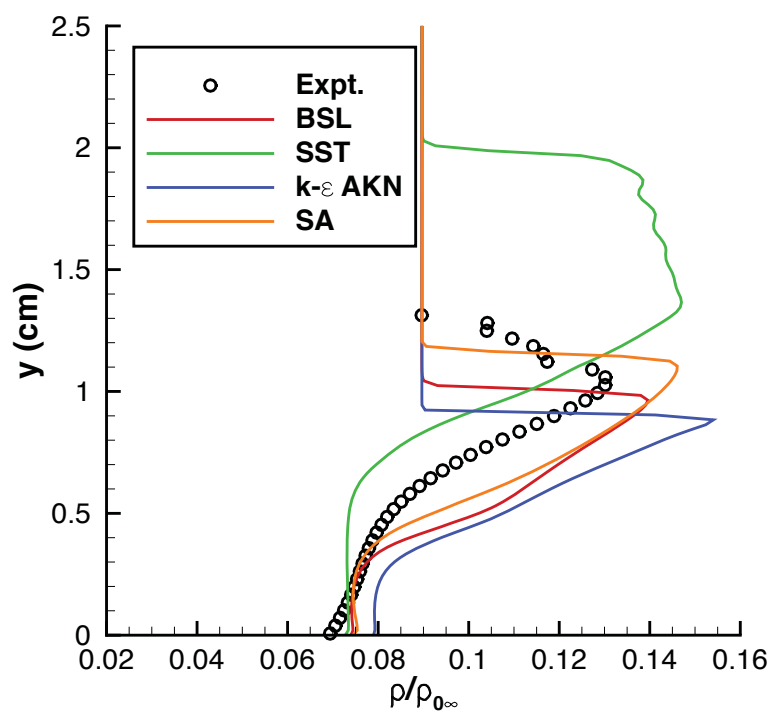

(d) $x=-1.007 \mathrm{~cm}$

Figure 10: Turbulence model comparison for density profiles, $\rho / \rho_{0 \infty}$ 


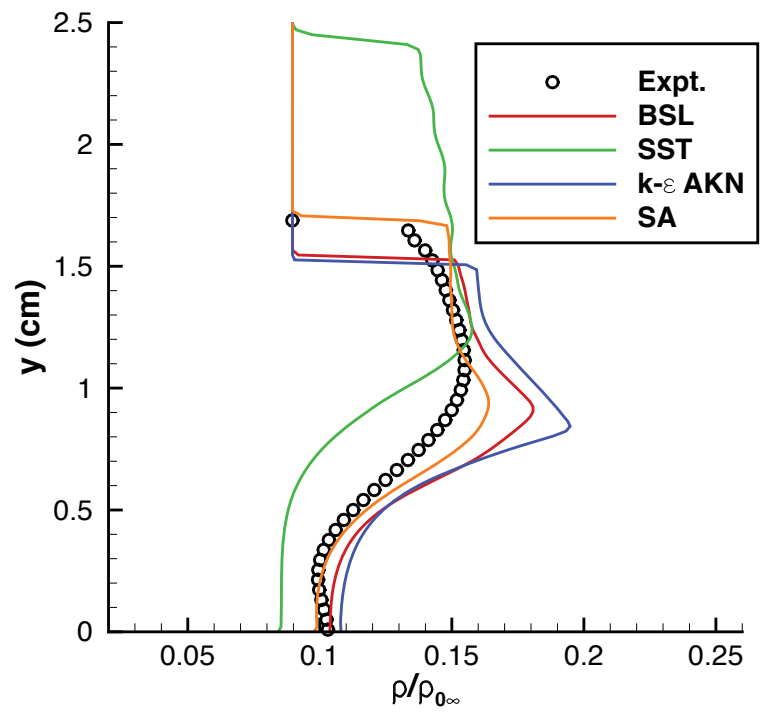

(e) $x=0.996 \mathrm{~cm}$

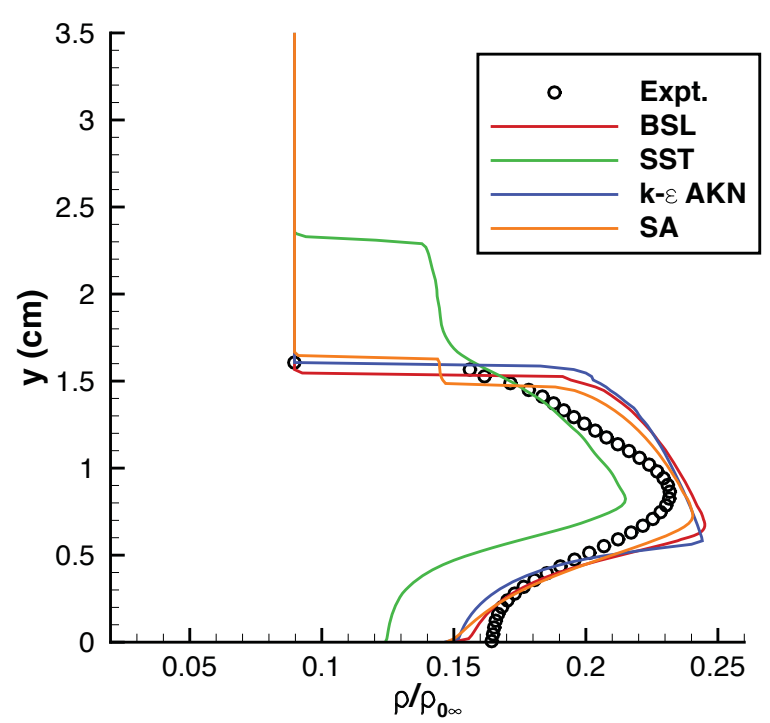

(g) $x=2.996 \mathrm{~cm}$

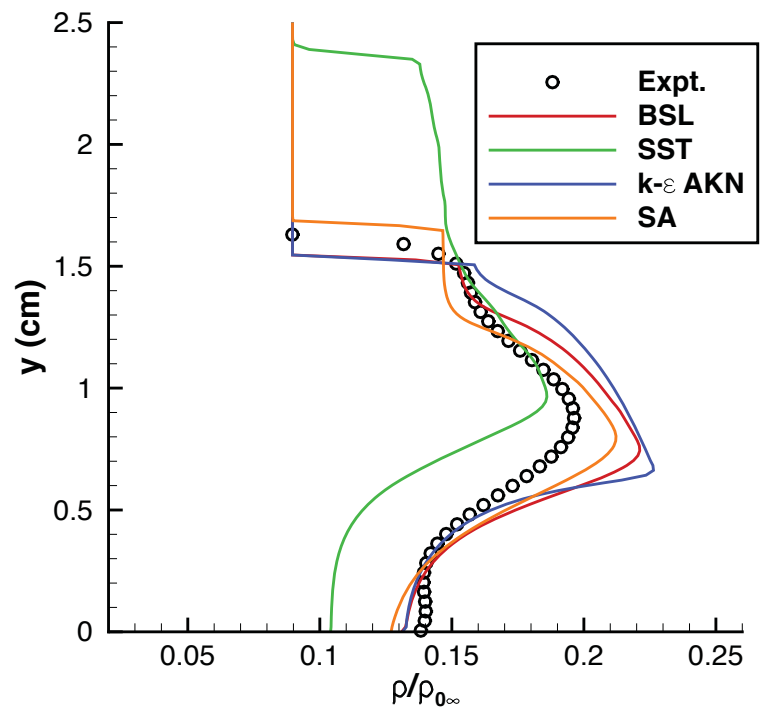

(f) $x=1.998 \mathrm{~cm}$

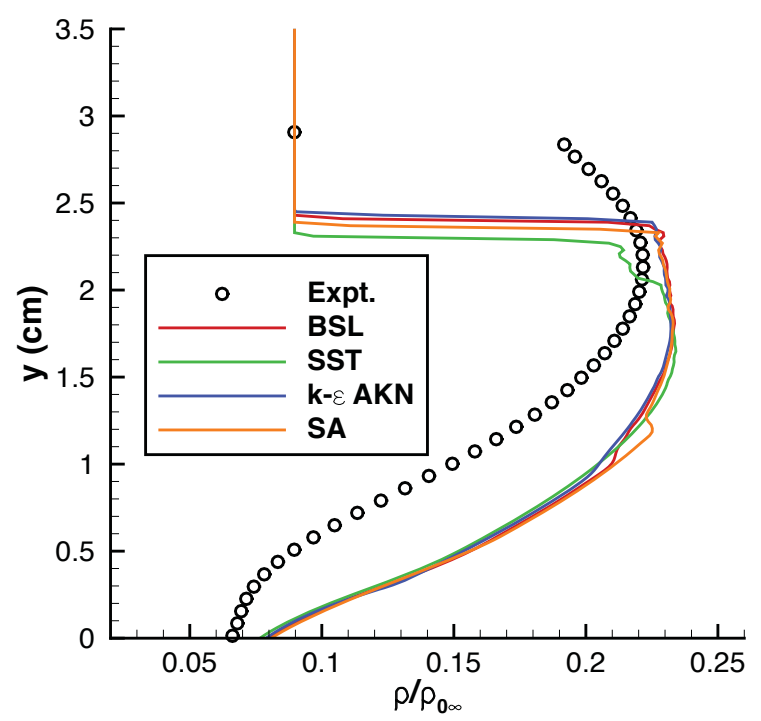

(h) $x=6.173 \mathrm{~cm}$

Figure 10: (continued) Turbulence model comparison for density profiles, $\rho / \rho_{0 \infty}$ 


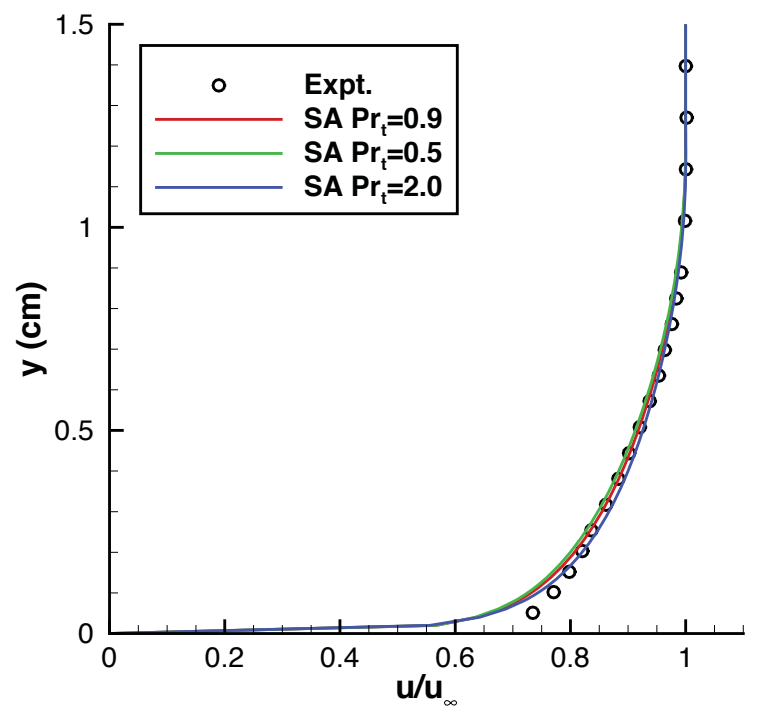

(a) Velocity profiles, $u / u_{\infty}$, at $x=-4.500 \mathrm{~cm}$

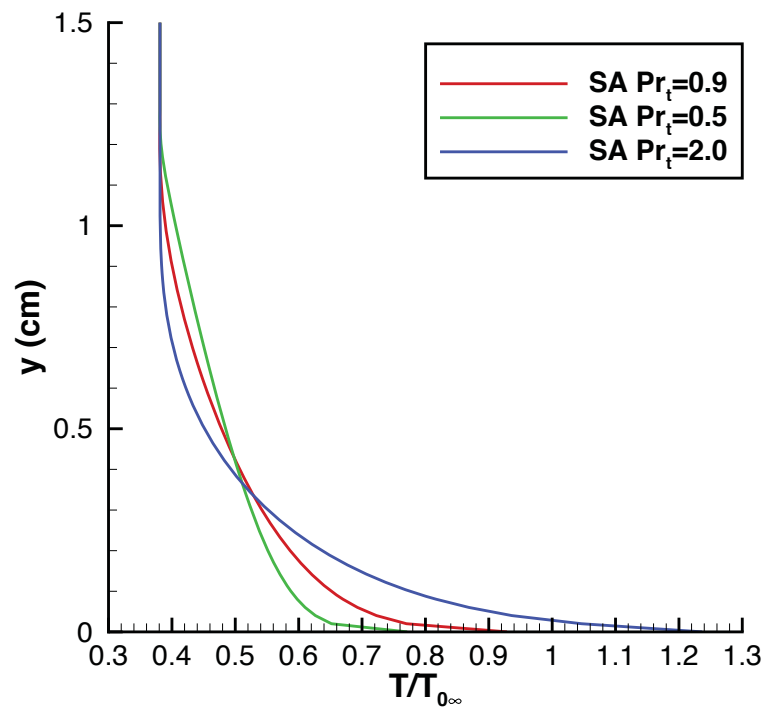

(b) Temperature profiles, $T / T_{0 \infty}$, at $x=-5.030 \mathrm{~cm}$

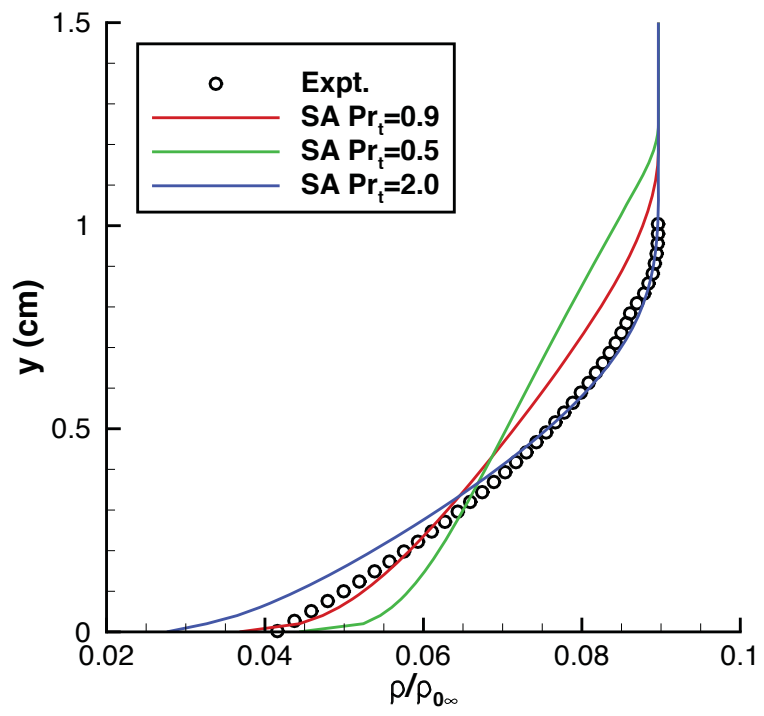

(c) Density profiles, $\rho / \rho_{0 \infty}$, at $x=-5.030 \mathrm{~cm}$

Figure 11: Effect of turbulent Prandtl number, $P r_{t}$, on upstream profiles 


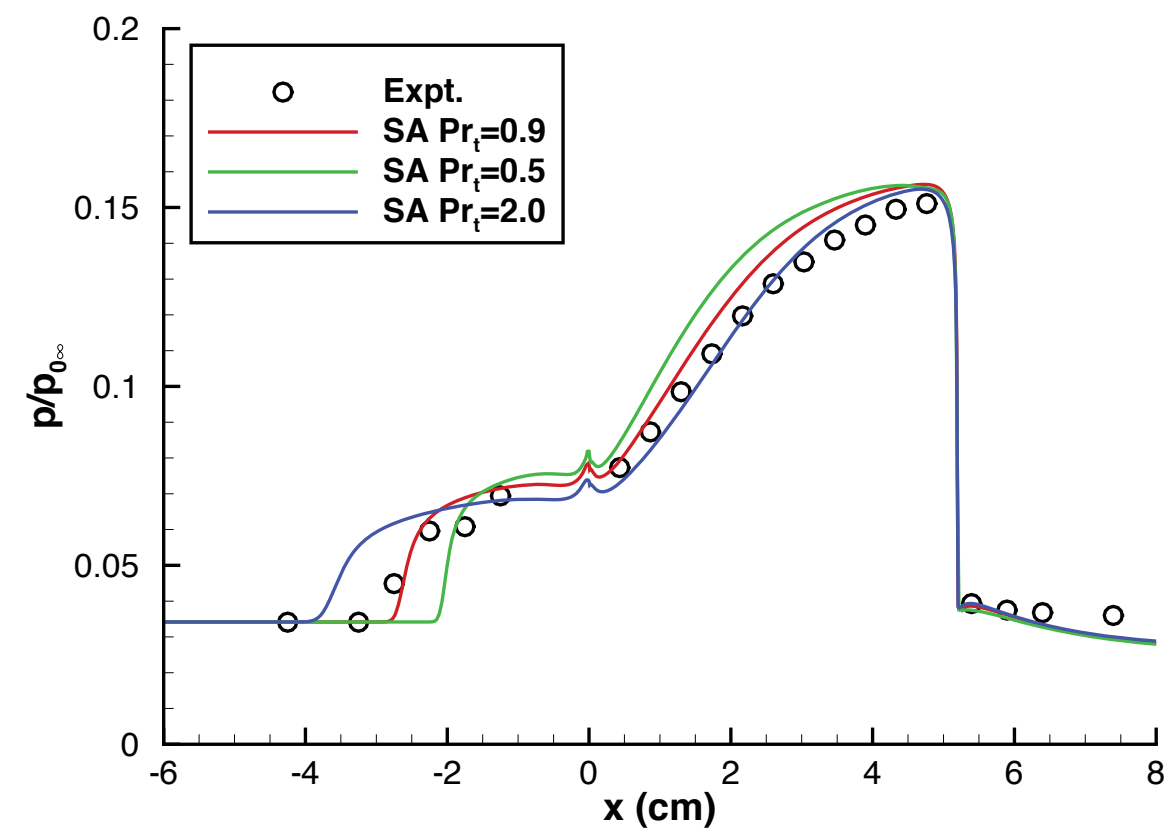

Figure 12: Effect of turbulent Prandtl number, $P r_{t}$, on surface static pressure coefficient, $p / p_{0 \infty}$

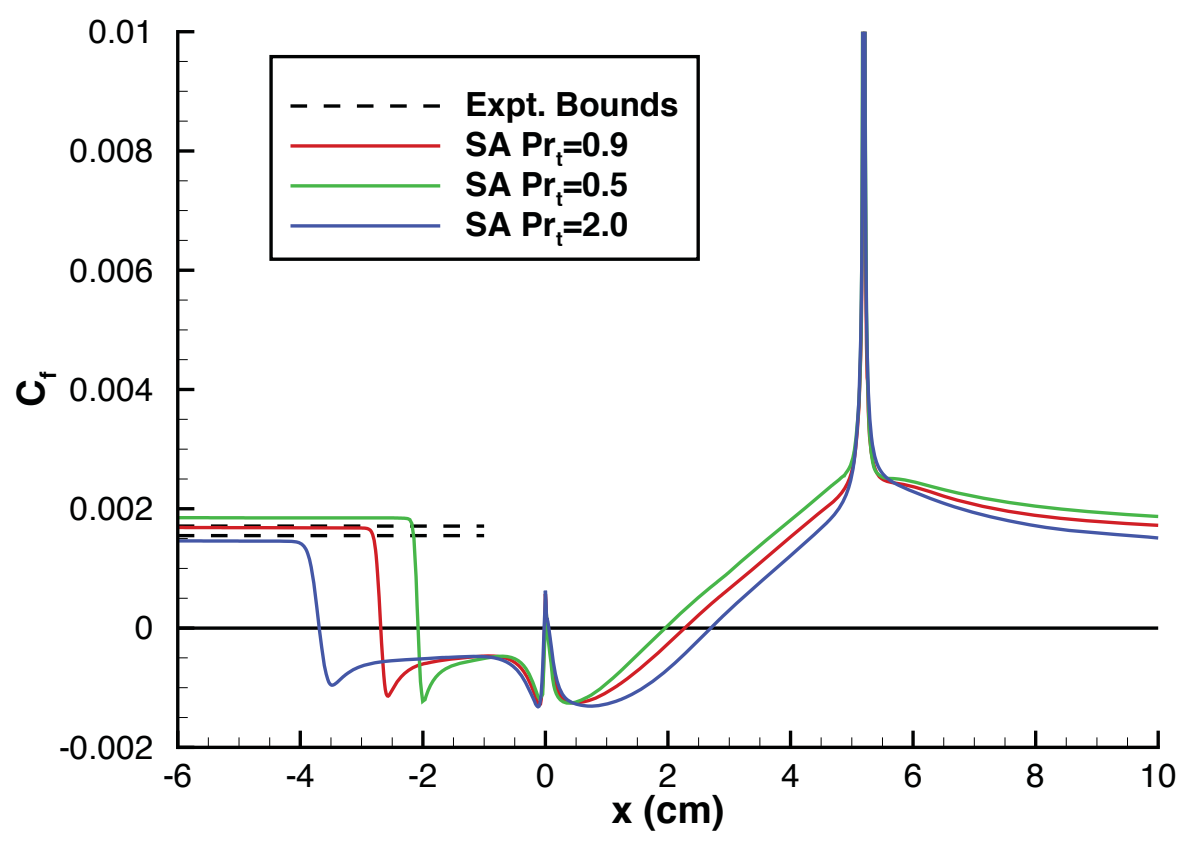

Figure 13: Effect of turbulent Prandtl number, $P r_{t}$, on skin friction coefficient, $C_{f}$ 


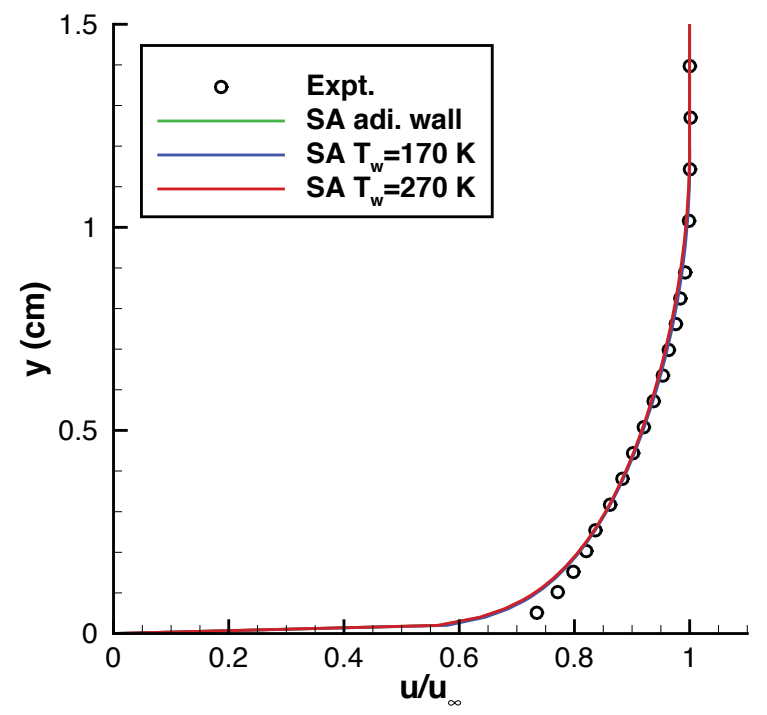

(a) Velocity profiles, $u / u_{\infty}$, at $x=-4.500 \mathrm{~cm}$

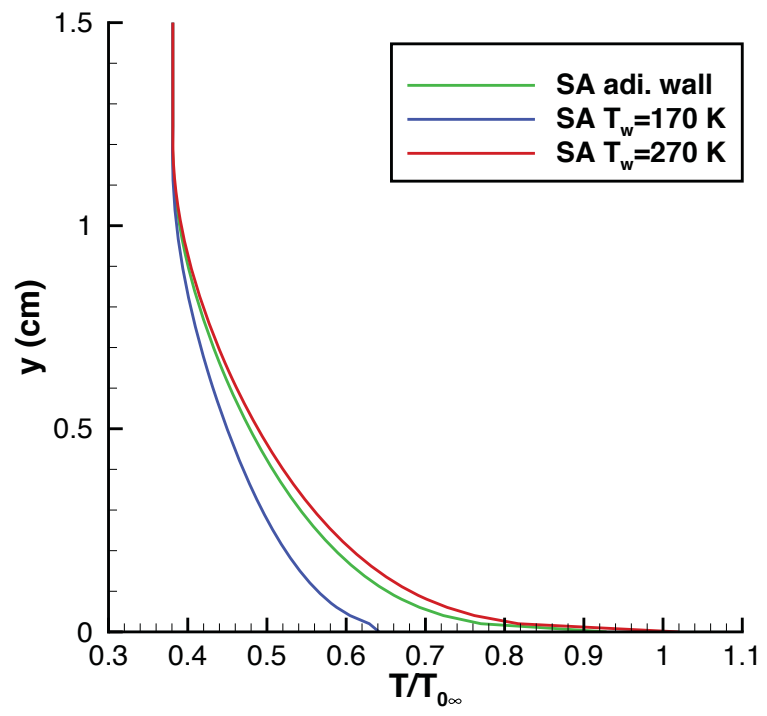

(b) Temperature profiles, $T / T_{0 \infty}$, at $x=-5.030 \mathrm{~cm}$

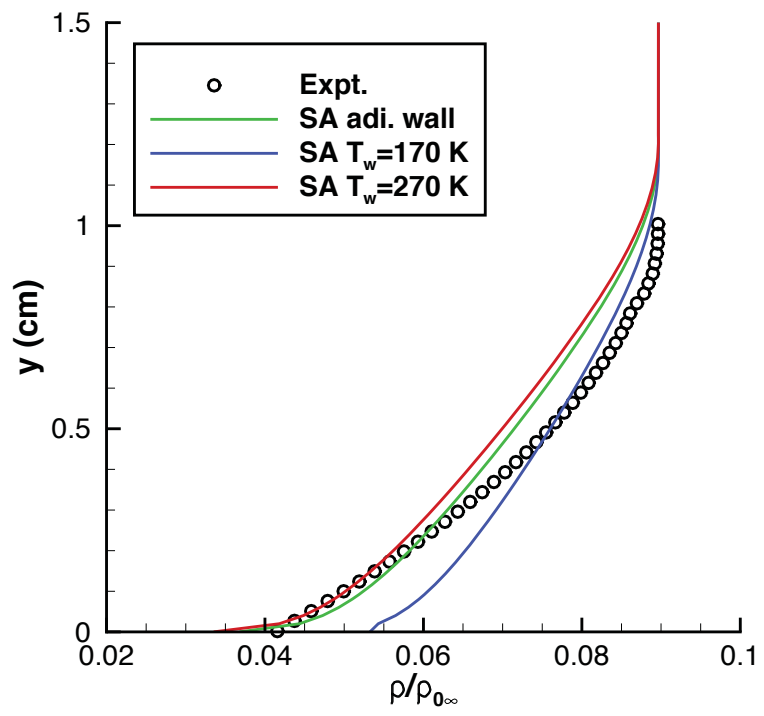

(c) Density profiles, $\rho / \rho_{\infty}$, at $x=-5.030 \mathrm{~cm}$

Figure 14: Effect of wall temperature, $T_{w}$, on upstream profiles 


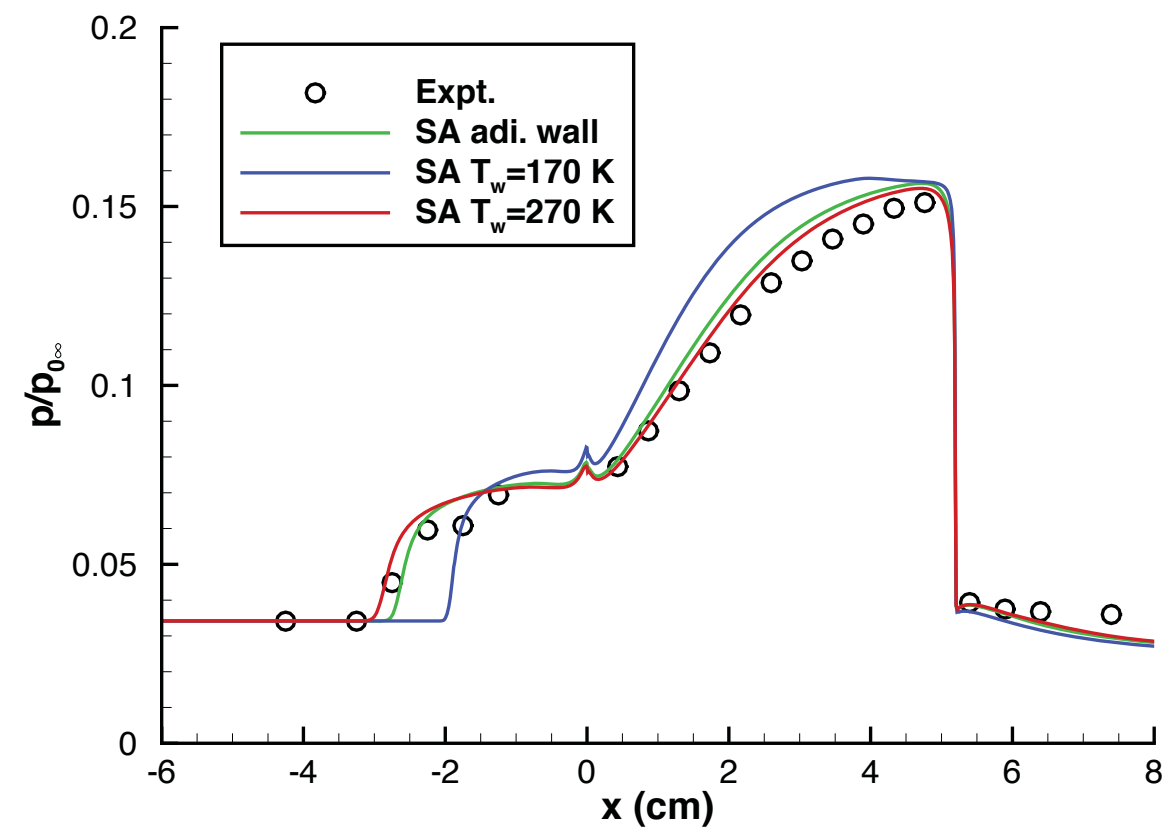

Figure 15: Effect of wall temperature, $T_{w}$, on surface static pressure coefficient, $p / p_{0 \infty}$

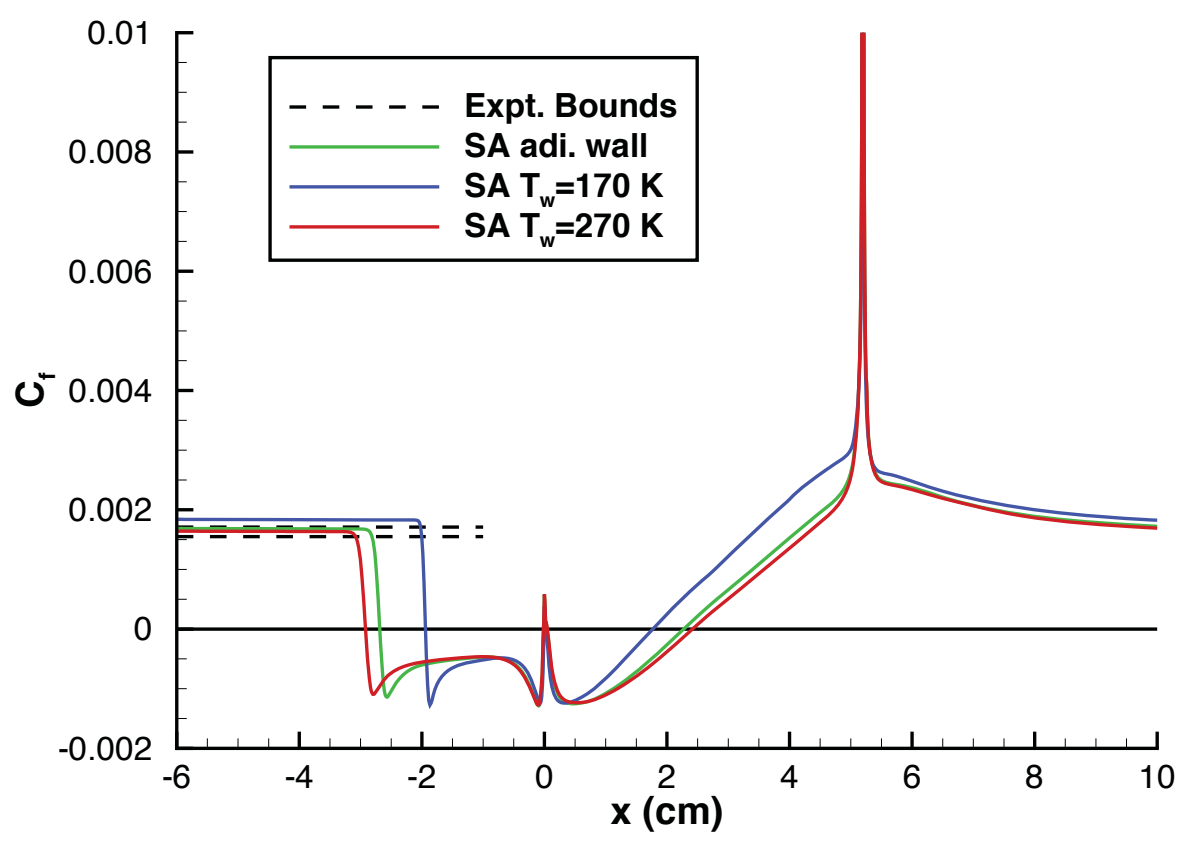

Figure 16: Effect of wall temperature, $T_{w}$, on skin friction coefficient, $C_{f}$ 